\title{
Rab27a and Rab27b control different steps of the exosome secretion pathway
}

Matias Ostrowski ${ }^{1,2}$, Nuno B. Carmo ${ }^{3^{*}}$, Sophie Krumeich ${ }^{1,2^{*}}$, Isabelle Fanget ${ }^{9^{*}}$, Graça Raposo $^{4,2}$, Ariel Savina ${ }^{1,2}$, Catarina F. Moita ${ }^{3}$, Kristine Schauer ${ }^{4,2}$, Alistair N. Hume ${ }^{5}$, Rui P. Freitas $^{3}$, Bruno Goud ${ }^{4,2}$, Philippe Benaroch ${ }^{1,2}$, Nir Hacohen ${ }^{8}$, Mitsunori Fukuda ${ }^{10}$, Claire Desnos $^{9}$, Miguel C. Seabra ${ }^{5,6,7}$, François Darchen ${ }^{9}$, Sebastian Amigorena ${ }^{1,2}$, Luis F. Moita ${ }^{3 \#}$ and Clotilde Thery ${ }^{1,2 \#}$.

${ }^{1}$ INSERM U932, 26 rue d’Ulm, 75005 Paris, France

${ }^{2}$ Institut Curie, 26 rue d’Ulm, 75005 Paris, France

${ }^{3}$ Cell Biology of the Immune System Unit, Instituto de Medicina Molecular Sala P3-B-40, Edifício Egas Moniz, Av. Prof. Egas Moniz, 1649-028 Lisboa; Portugal

${ }^{4}$ CNRS UMR144, 26 rue d’Ulm, 75005 Paris, France

${ }^{5}$ Molecular Medicine, National Heart and Lung Institute, Imperial College London, London UK

${ }^{6}$ CEDOC, Faculdade de Ciências Médicas, Universidade Nova de Lisboa, Lisboa, Portugal ${ }^{7}$ Instituto Gulbenkian de Ciência, Oeiras, Portugal

${ }^{8}$ Center for Immunology and Inflammatory Diseases, Division of Rheumatology, Allergy, and Immunology, Massachusetts General Hospital, Charlestown, MA, USA and Broad Institute of Harvard and MIT, Cambridge, MA, USA.

${ }^{9}$ Institut de Biologie Physico-chimique, Centre National de la Recherche Scientifique, FRE 3146, 13 rue Pierre et Marie Curie, 75005 Paris

${ }^{10}$ Laboratory of Membrane Trafficking Mechanisms, Department of Developmental Biology and Neurosciences, Tohoku University, Miyagi, Japan

*: these authors contributed equally

\#: corresponding authors. Clotilde.thery@curie.fr; lmoita@fm.ul.pt. 


\section{ABSTRACT}

Exosomes are secreted membrane vesicles that share structural and biochemical characteristics with intraluminal vesicles of multivesicular endosomes (MVEs). Exosomes could be involved in intercellular communication and in the pathogenesis of infectious and degenerative diseases. The molecular mechanisms of exosome biogenesis and secretion are, however, poorly understood. Using a RNA interference screen, we identified five Rab GTPases that promote exosome secretion in HeLa cells. Among these, Rab27a and Rab27b were found to act in MVE docking at the plasma membrane. The size of MVEs was strongly increased by Rab27a silencing, whereas MVEs were redistributed towards the perinuclear region upon Rab27b silencing. Thus, the two Rab27 isoforms play different roles in the exosomal pathway. In addition, silencing two known Rab27 effectors, Slp4 (SYTL4) and Slac2b (EXPH5), inhibited exosome secretion and phenocopied silencing of Rab27a and Rab27b, respectively. Our results therefore strengthen the link between MVEs and exosomes, and open ways to manipulate exosome secretion in vivo. 


\section{INTRODUCTION}

Exosomes are membrane vesicles secreted into the extracellular space by numerous cell types $^{1-4}$. These nanometer-sized vesicles have a similar membrane orientation as the plasma membrane, and a canonical protein composition, mostly including proteins from the endocytic system, the plasma membrane and cytosol. In addition, exosomes also contain proteins specific of the cell type from which they originate and that are probably important for their extracellular functions. Based on in vitro studies, it has been proposed that exosomes could participate in induction of immune responses ${ }^{5-7}$, in the dissemination of viruses or prions ${ }^{8,9}$, in the pathogenesis of neurodegenerative diseases ${ }^{10}$, and in mediating communication between tumor cells and their microenvironment ${ }^{11-13}$. However, conclusive demonstration that in vivo exosome secretion plays a role in any physiological process has not been provided so far, due to the lack of tools allowing to specifically inhibit or increase their secretion. Indeed, the molecular mechanisms of exosome secretion are not completely understood.

Evidence collected during the last 20 years suggests that in most cell types, exosomes correspond to secreted intraluminal vesicles (ILVs) of MVEs ${ }^{4}$. ILVs are formed by inward budding of the limiting membrane of MVEs into the lumen of these endosomes ${ }^{14}$. The observation by electron microscopy (EM) of fusion profiles between MVEs and the plasma membrane, with the consequent release of ILVs as exosomes, has been to date the strongest evidence for the endosomal origin of exosomes ${ }^{5,6,15}$. Nevertheless, in some cell types such as $\mathrm{T}$ lymphocytes, exosomes bud at the plasma-membrane from endosome-like domains ${ }^{16}$. Therefore, identification of molecules that control the intracellular trafficking of MVEs and their fusion with the plasma membrane will improve our understanding of exosome secretion. Most intracellular transport pathways are controlled by conserved families of cytosolic proteins, including the Rab family of small GTPases. Rab proteins control different steps of vesicular trafficking, including budding, motility, docking to and fusion of different vesicular transport intermediates with acceptor membranes ${ }^{17}$. Almost 70 Rabs and Rab-like proteins have been identified in humans ${ }^{18}$, and a wide range of specific effector proteins that execute their diverse roles have also been identified ${ }^{19}$.

We analysed here the roles that Rab proteins could play in exosome production or secretion by the human HeLa cell line, using an shRNA-based screen targeting human Rabs. We observed that knocking-down five Rab proteins (Rab2b, Rab9a, Rab5a, Rab27a and Rab27b) inhibited exosome secretion without major modifications in the secretion of soluble proteins 
through the regular secretory pathway. The roles played by Rab27a and Rab27b were analysed further, and we evidenced both a common function in MVE docking to the plasma membrane, but also different and non-redundant roles in the MVE pathway. In addition, silencing of two known effectors of Rab27, Slp4 (synaptotagmin-like 4 = SYTL4) and Slac2b (exophilin 5 = EXPH5), phenocopied silencing of, respectively, Rab27a and Rab27b, suggesting a preferential interaction between these proteins for exosome secretion. 
RESULTS

\section{Semiquantitative detection of exosomes in cell culture supernatants}

To screen molecules specifically involved in exosome secretion, we developed a semiquantitative fluorescence-activated cell scanning (FACS)-based assay allowing simultaneous detection of exosomes and of proteins secreted through the classical secretory pathway, in small volumes of cell culture supernatants. The HeLa B6H4 tumor cell line, which stably expresses the transactivator CIITA (driving expression of the MHC class II family genes, including HLA-DR) and a secreted form of chicken ovalbumin (OVA), was used. These cells secrete HLA-DR-positive exosomes and soluble OVA (secreted through the classical secretory pathway), allowing differentiation of both pathways. Exosomes present in cell culture supernatants were captured onto beads coated with antibodies to CD63, a tetraspanin strongly enriched in late endosomes and exosomes ${ }^{20}$, and detected by FACS after staining with anti-HLA-DR and anti-CD81 fluorescent antibodies (CD81 is another tetraspanin abundant on exosomes) ${ }^{5,20}$, or Annexin V (which binds to the phosphatidylserine (PS) present at the surface of exosomes) ${ }^{21}$ (Fig. 1a). Membrane vesicles with a diameter ranging from 50 to $100 \mathrm{~nm}$ and with the typical cup-shaped morphology of exosomes were detected by electron microscopy (EM) on the surface of the beads (Fig. 1b). Exosome detection was dependent on the number of exosome-secreting cells present in the wells (Fig. 1c, d), with a threshold of sensitivity of 75,000 cells/well. When supernatants from apoptotic cells (which secrete apoptotic blebs but not exosomes ${ }^{22}$ ) were used, the FACS signals were significantly lower than those obtained from supernatants of living cells (Fig. 1e).

Secretion of soluble OVA in cell culture supernatants was detected using beads coated with anti-OVA antibodies and stained with a fluorescent anti-OVA antibody (Supplementary information, Fig. S1). This methodology allowed the quantitative detection of OVA from live cells, with a sensitivity of 30,000 cells/well.

This FACS-based assay thus allows simultaneous quantification of CD63/CD81/HLADR/PS-positive membrane vesicles (corresponding to exosomes) and of OVA produced by living cells in very small volumes of culture supernatants collected from 96-well plates.

\section{Role played by members of the Rab GTPase family in exosome secretion}


A lentiviral shRNA library targeting 59 members of the Rab GTPase family was screened using the methodology described above as readout. Cells expressing scrambled shRNA were used as a control of both OVA and exosome secretion pathways (Fig. 2a). Genes for which at least two different shRNAs induced a significant modification in the secretion of exosomes, but not of OVA, were selected for two additional rounds of validation.

Some examples of Rab proteins that did not fulfill the selection criteria are shown in Fig. $2 \mathrm{~b}$. Silencing of Rab6a inhibited the secretion of OVA but did not affect exosome secretion, whereas inhibition of Rab7 or Rab11a modified neither exosome nor OVA secretion significantly. In contrast, five Rab proteins whose inhibition by shRNA resulted in decreased exosome secretion in three independent experiments, and for which most shRNA modified concomitantly the phenotype and the mRNA expression level (as quantified by qPCR), were finally selected (Fig. 2c): Rab2b, Rab5a, Rab9a, Rab27a and Rab27b. Most of these Rabs have been previously associated with endocytic functions, in agreement with the postulated endosomal origin of exosomes.

We chose to further analyze the mechanism of inhibition of exosome secretion by Rab27a and Rab27b, two homologous Rab proteins (71\% amino acid identity ${ }^{23}$ ) encoded by different genes, and associated with lysosome-related organelles. To ensure that Rab27 silencing affects total exosome secretion rather than any specific exosome-associated protein, we performed annexin V staining of PS on exosomes captured by CD63-coated beads (Fig. 2d). Silencing of both Rab27a and Rab27b decreased annexin V staining, thus showing a general decrease of secretion of exosome-associated lipids.

\section{Rab27a and Rab27b silencing inhibit exosome secretion without modifying their protein composition}

Inhibition of exosome secretion in cells knocked down (KD) for Rab27a and Rab27b was confirmed by standard techniques of exosome purification ${ }^{24}$. First, cells transfected with shRNA\#3 for Rab27a or shRNA\#1 for Rab27b were amplified to produce a large volume of supernatant. Expression of the respective genes was specifically and significantly downregulated in these cells (supplementary information, Fig. S2a). Exosomes were purified from cell supernatant by differential ultracentrifugation ${ }^{24}$. The total amount of secreted exosomes, determined by measuring total amount of proteins in the 100,000g pellet (Fig. 3a), or by the FACS-based assay (Fig. S2b), was significantly reduced in both KD cells, as compared to control cells. 
Consistently, a reduction in the signals of several exosome markers (HLA-DR, CD63, tsg101, and hsc70) was observed by Western blotting performed on exosomes purified from the supernatant of the same number of Rab27a-KD or Rab27b-KD secreting cells, as compared to control cells (Fig. 3b and FigS6a). Nevertheless, all markers were detected in exosomes from KD cells when similar amounts of exosomal proteins where analysed (Fig. 3c and Fig.S6b). In addition, exosomes secreted by Rab27a- and Rab27b-KD cells showed identical size and morphology, as assessed by EM, as exosomes produced by control cells (Fig. 3d).

These data show that silencing of either Rab27a or Rab27b reduces the amount of exosomes released in the cell-culture supernatant, but modifies neither their protein content nor their morphology.

\section{Different phenotypes of MVEs are observed in Rab27a and Rab27b KD cells}

To gain further insight into the mechanism responsible for the reduced exosome secretion in Rab27a and Rab27b KD cells, the subcellular distribution and the morphology of MVEs were investigated using CD63 as a marker. We first observed by FACS analysis that the proportion of intracellular vs. surface CD63 was increased in Rab27a, but not Rab27b, KD cells, as compared to control cells (Fig. 4a). Thus, in the absence of Rab27a, CD63 accumulates in internal compartments, whereas the absence of Rab27b does not induce such accumulation.

The subcellular distribution of CD63 was then analysed by immunofluorescence imaging (Fig. 4b, c). In control and Rab27a KD cells, CD63-positive MVEs appeared homogenously distributed throughout the cell, but their size was significantly increased upon Rab27a silencing (Fig. 4b upper and middle panels, Fig. 4c). In sharp contrast, Rab27b KD cells displayed a characteristic asymmetrical perinuclear accumulation of CD63-positive organelles, but without striking alteration of the size of these organelles, as compared to control cells (Fig. 4b lower panel, Fig. 4c). Consistently, EM analysis showed that the MVEs from Rab27a KD cells were significantly larger (Fig. 4d, right panel) than those from control cells, whereas in Rab27b KD cells, MVEs were smaller (Fig. 4d) and showed a clustered distribution (not shown). Thus, although silencing of either Rab27a or Rab27b impairs exosome secretion, the induction of different changes in the size and distribution of CD63positive MVEs suggests that both proteins play different roles in the pathway.

\section{Rab27a and Rab27b have different subcellular localizations}


We thus asked whether these two proteins could function in different compartments, by analyzing their respective subcellular localizations. GFP-tagged versions of both Rab27a and Rab27b presented a donut-like pattern surrounding CD63-positive compartments (Fig. 5a). However, most of Rab27b, but not of Rab27a, presented a perinuclear distribution, mainly concentrated in a perinuclear area labeled by the Golgi-associated proteins Rab6 (Fig. 5a) and TGN46 (not shown). Consistently, upon EM observation, although both Rab27a and Rab27b were associated with the limiting membrane of MVEs, Rab27b was also observed in the membrane of Golgi stacks and in vesicles located in the TGN area (Fig. 5b).

When mCherry-Rab27a and GFP-Rab27b were expressed simultaneously in HeLa cells, both Rab27a and Rab27b were partially associated with CD63-positive compartments (Fig. 5c), but Rab27b was predominantly in the perinuclear area with partial colocalization with TGN46 (data not shown). Four groups of CD63-positive organelles could be identified based on their association with the Rab27 isoforms: most organelles were Rab27a $/ \mathrm{b}^{-}$, about 1/5 were Rab27a ${ }^{+} / b^{-}$, and less than $10 \%$ were either $R a b 27 a^{+} / b^{+}$, or Rab27a $/ b^{+}$. However, the proportion of Rab27-positive MVEs increases in the subplasmalemmal region (see below).

Rab27a and Rab27b therefore have different subcellular distributions: Rab27b predominantly presents an asymmetrical perinuclear distribution in the TGN area and a minor localization onto CD63-positive compartments, whereas Rab27a is preferentially associated with CD63positive MVEs.

\section{Rab27a or Rab27b silencing reduces MVE docking to the plasma membrane}

To further analyze exosome secretion, we used total internal reflection fluorescence microscopy (TIRFM) to image MVEs in the subplasmalemmal region of live cells (Fig. 6, Supplementary movies 1-3, Fig. S3). In this region, around 30\% and 40\% of CD63-positive vesicles were labeled with GFP-Rab27b and GFP-Rab27a, respectively (Fig. S3a), supporting a possible role of both Rab27 isoforms in MVE exocytosis. Rab27b silencing significantly lowered MVE density (Fig. 6a), in agreement with the perinuclear relocalization of MVEs observed by epifluorescence microscopy (Fig. 4b). In addition, in Rab27a and Ra27b KD cells, MVEs were significantly less present near the borders of the cell footprint (Fig. S3b), suggesting that Rab27a and Rab27b promote their distribution at the cell periphery.

The movements of MVEs were then analysed by tracking individual trajectories ${ }^{25}$ (Supplementary movies 1-3). The diffusion coefficient $\left(D_{x y}\right)$, an index of mobility, was calculated along trajectories using a rolling analysis window ${ }^{25} . D_{x y}$ values were higher in 
Rab27a- or Rab27b-KD cells than in controls ( $\mathrm{p}<0.0001$ ), and in Rab27b-KD than in Rab27aKD cells $(\mathrm{p}=0.006)$ (Fig. 6b). Fewer vesicles had very low $D_{x y}$ values in Rab27a-KD or Rab27b-KD cells, suggesting that both may participate in MVE attachment (docking) at the plasma membrane, a process that restricts vesicle motion ${ }^{25-27}$. To set a threshold value below which vesicles could be considered as immobile, we plotted the distribution of instantaneous $D_{x y}$ values (Fig. S3c). A major peak comprising the lowest values was centered around $10^{-4}$ $\mu \mathrm{m}^{2} /$ s (i.e. the mean $D_{x y}$ of immobilized beads on our setup ${ }^{26}$ ). Vesicles presenting a $D_{x y}$ less than three times this value were considered as immobile. Using this criterion, we categorized $D_{x y}$ values computed on entire trajectories and found that the percentage of docked structures was significantly lowered by Rab27a and Rab27b silencing (Fig. 6c). Moreover, $82 \%$ of the enlarged MVEs observed upon Rab27a silencing (see Fig. 4b) displayed a mean $D_{x y}>3 \times 10^{-4}$ $\mu \mathrm{m}^{2} . \mathrm{s}^{-1}$ (50 structures from 5 cells) and were thus not docked.

To distinguish bona fide vesicle docking from other processes, we measured the duration of immobilization events along trajectories. Rab27a and Rab27b silencing reduced the percentage of vesicles that were always immobile and increased the percentage of vesicles never immobile (Fig 6d). Survival curves of immobilization times were best fitted with two exponentials, indicating the existence of two different processes (Fig. 6e). The short-lasting component may reflect transient tethering to the plasma membrane or phases of slow diffusion. We considered that long-lasting immobilization reflected MVE docking. Using this refined criterion of docking, we conclude that Rab27a and Rab27b silencing reduced both the number (0.25 and 0.28 docking event/trajectory in Rab27a and Rab27b KD cells, respectively, compared to 0.44 in controls) and the characteristic time (10.1, 9.3 and $15.5 \mathrm{~s}$ in Rab27a-, Rab27b-KD cells and controls, respectively) of docking events. Altogether, these analyses show that Rab27a and Rab27b promote the occurrence and stability of MVE docking.

Another alteration was observed in Rab27b-KD cells. The proportion (39 \pm 3\% vs $29 \pm 3 \%$ in control cells) and velocity (Fig. 6f) of vesicles moving rapidly along linear axes (a characteristic of microtubule-based motion ${ }^{26}$ ) were increased in Rab27b-KD (but not in Rab27a-KD) cells, as compared to control ones. This behavior accounts for the increased proportion of structures with high mean $D_{x y}\left(>20 \times 10^{-4} \mu^{2}{ }^{2} \mathrm{~s}^{-1}\right)$ (Fig. 6c) observed in Rab27b KD. Therefore, Rab27b also negatively regulates long-range movements of MVEs.

\section{Slp4 and Slac2b are two Rab27 effectors involved in exosome production}


Rab proteins exert their functions by interacting with multiple effectors. We observed that 7 of the 11 Rab27 effector proteins were expressed in HeLa B6H4 cells (supplementary Table 1). These genes were then silenced with specific shRNAs and quantification of exosomes and OVA was performed in cell culture supernatants using the FACS-based assay.

Silencing of Slac2b (or exophilin-5, EXPH5), Slp4 (or synaptotagmin-like 4, SYTL4) and Munc13-4 significantly reduced exosome secretion, in at least 2 of the 4-5 shRNAs that target each gene (Fig. 7a). In the cells transduced with shRNAs against Slp4 and Slac2b, the level of exosome secretion paralleled the level of mRNA expression (Fig. 7b). In contrast, in the case of Munc13-4, although shRNAs \#1, 2 and 3 decreased both exosome secretion and mRNA levels, shRNAs 4 and 5 strongly inhibited mRNA expression without affecting exosome secretion (Fig. 7a,b), suggesting off-target effects of the shRNA. Consequently, only Slac2b and Slp4 were chosen for further analysis, although a role of Munc13-4 in exosome secretion cannot be ruled out. In addition to reducing exosome secretion, knock down of the effectors increased OVA secretion (Fig. 7a), suggesting that they also play Rab27-independent roles in the conventional secretion pathway.

Large-scale cultures of cells expressing lentiviruses \#3 (Slac2b) or \#3 (Slp4) where set up. Specific decrease of Slp4 at the protein level was confirmed by immunoblotting (Fig. 7c and FigS6c), but we could not specifically detect Slac2b by Western blot in B6H4 cell lysates, probably due to low level of expression. Exosomes secreted by the same amount of cells were purified from cell culture supernatants and analysed by western blot (Fig. 7d and and FigS6e): a significant reduction in the amount of CD63, HLA-DR and tsg101 secreted in exosomes was observed in Slp4- and Slac2b-KD cells. These results show that two Rab27 effectors, Slp4 and Slac2b, are positive regulators of exosome secretion in HeLa B6H4 cells.

\section{Slp4 and Slac2b KD are phenocopies of Rab27a and Rab27b KD cells, respectively}

The distribution and morphology of CD63-positive compartments was then studied in Slac2band Slp4-KD cells. In Slp4-KD cells, an intracellular accumulation of CD63 was observed both by FACS (Fig. 7e) and by immunofluorescence (Fig. $7 \mathrm{f}$ left and middle panels), resembling the phenotype observed in Rab27a KD cells (Fig. 4a). In contrast, Slac2b-KD cells did not show an accumulation of CD63 either at the cell surface or intracellularly by FACS, but exhibited an asymmetrical perinuclear accumulation of CD63-positive vesicles of normal size, mimicking the phenotype observed in Rab27b KD cells (Fig. 7f, right panel). Altogether, loss of function of Slp4 and Slac2b induces phenotypes that, in terms of exosome 
secretion as well as morphology and distribution of MVEs, highly resemble those observed in cells KD for Rab27a and Rab27b, respectively. These observations suggest that Slp4 mediates the function of Rab27a, while Slac2b is required to achieve Rab27b’s function.

Supporting this idea, in cells co-transfected with T7-tagged Slp4 or -Slac2b and GFP-tagged Rab27a or Rab27b, Slp4 colocalized with Rab27a on donut-like structures containing CD63 (Fig. 8a, top panel). In contrast, Slp4 and Rab27b labeled different CD63-positive structures (Fig. 8a, bottom panel). The Rab27a/Slp4 interaction was also confirmed by the observation that Slp4 protein level was decreased in Rab27a KD (but not in Rab27b KD) (Fig. 8b and Fig. S6d), whereas its mRNA level was increased (Fig. 8c), suggesting a stabilizing interaction of Rab27a and Slp4 proteins. Due to low level of expression of endogenous Slac2b, which could not be detected by the available antibodies, and to poor expression of a T7-Slac2b construct in our cell line, we could neither determine its intracellular distribution nor its preferential association with Rab27b. Finally, co-silencing of Rab27a and Slp4 led to a similar phenotype as single silencing of each (i.e. enlarged MVEs), whereas co-silencing of Rab27b and Slp4 induced mainly the perinuclear redistribution of MVEs typical of Rab27b-KD cells, thus confirming that Rab27b and Slp4 are not functionally associated (Fig. S4).

These observations thus show that Rab27a and Slp4 function together in the MVE and exosome pathway of HeLa cells. By contrast, although silencing of Slac2b induces a phenocopy of Rab27b-KD cells, we could not demonstrate, for technical reasons, the functional interaction between these two proteins. 


\section{DISCUSSION}

Three major results are presented here. First, we have set up an assay allowing medium-scale screens of the molecular machinery involved in exosome secretion. Second, using this assay, we identified several Rab family members, including Rab27a and Rab27b, that play a role in exosome secretion. Third, we found that Rab27a and Rab27b serve both common and different roles, most likely via the Slp4 and Slac2b effector proteins respectively, in the intracellular trafficking of multivesicular endosomes leading to exosome secretion.

The identification of Rab GTPases that affect both the morphology and distribution of CD63positive MVEs and exosome secretion confirms the endocytic origin of exosomes, previously proposed by elegant EM studies ${ }^{5,15}$. In addition, since reducing mRNA expression of Rab27 genes to less than $20 \%$ of the control induced over $50 \%$ reduction of exosome secretion, the majority of secreted exosomes originate from a Rab27-dependent secretion event, probably due to fusion of late endocytic compartments with the plasma membrane. In addition to Rab27, three other Rabs related to the endocytic pathway were identified in this study as potentially involved in exosome secretion: Rab9 and Rab5 participate in the trafficking of late and early endosomes, respectively ${ }^{17,28}$ and Rab2, although generally described between endoplasmic reticulum and Golgi apparatus, is phylogenetically closer to Rab4, Rab11 and Rab14 ${ }^{29}$, which are associated with the endocytic pathway. Surprisingly, other Rabs associated with the endocytic system, Rab7 or Rab11, did not modify exosome secretion. These observations suggest that a specific subset of MVEs participates in the generation of exosomes, as recently proposed in dendritic cells ${ }^{30}$.

Rab27a and Rab27b have been previously involved in the regulated secretion of secretory granules (i.e. compartments of Golgi origin) ${ }^{31-33}$ and of lysosome-related organelles in mast cells ${ }^{34}$, platelets ${ }^{35}$, and cytolytic $\mathrm{T}$ lymphocytes ${ }^{36}$. In this study, we found that Rab27a and Rab27b play a key role in exosome secretion by promoting the targeting of MVEs to the cell periphery and their docking at the plasma membrane.

Functional redundancy between Rab27a and Rab27b depends on the cell type and/or the secretory process studied ${ }^{34,35,37}$. Interestingly, we show here that in the HeLa cell line, Rab27a and Rab27b perform different and non-redundant tasks in the exosomal pathway.

The phenotype of MVEs induced by Rab27b silencing (inhibition of docking, clustering in the perinuclear area and high velocity movements along microtubules), is similar to the phenotype of secretory granules ${ }^{31}$ and melanosomes ${ }^{38,} 39$ induced by silencing Myosin Va, a 
downstream effector of Rab27a, and suggests that Rab27b mediates the transfer of MVEs from microtubules to the actin-rich cortex and their retention at the cell periphery. In addition, the smaller size of MVEs observed by EM in Rab27b KD cells suggests that Rab27b could participate in the transfer of membranes from TGN to MVEs, as previously proposed ${ }^{40}$.

In contrast, Rab27a silencing reduces docking of MVEs and increases their size. This could be due to lack of motor linkage, resulting in apparently enlarged compartments. Alternatively, either Rab27a is required for docking and vesicles fuse with each other instead of fusing with the plasma membrane when Rab27a is absent, or Rab27a prevents fusion of CD63-positive compartments with each other or with other vesicular intermediates, and its absence leads to the formation of enlarged compartments whose size physically impairs docking to the plasma membrane.

In addition, we show here that silencing of the Rab27 effectors Slp4 and Slac2b phenocopies the silencing of Rab27a and Rab27b, respectively, and that Slp4 preferentially associates with Rab27a, rather than with Rab27b, and thus regulates the Rab27a-dependent docking event leading to exosome secretion in HeLa cells. The preferential interaction of Rab27b with Slac2b in HeLa cells could not be demonstrated here, although it has been previously demonstrated in vitro ${ }^{41}$.

Finally, we must stress that this work was performed using the HeLa model cell line: the physiological processes at work in HeLa may not be identical to those of other cell types. However, mouse dendritic cells deficient for both Rab27a and Rab27b secrete half the amount of exosomes than their wild-type counterparts (Fig. S5) and our preliminary data suggest that silencing of Rab27a in a mouse tumor cell line also leads to reduced exosome secretion (A. Bobrie, M.O. and C.T., unpublished data). Thus, the Rab27 subfamily is probably also important in exosome secretion by other cell types.

Overall, the possibility to specifically inhibit exosome secretion without affecting the regular secretory pathway of soluble proteins paves the way to manipulate their secretion in vivo, to eventually answer the long-lasting question of the physiological functions played by exosomes in vivo.

\section{ACKNOWLEDGEMENTS}


This work was supported by post-doctoral salaries from Institut National du Cancer and Institut Curie to M.O., grants from Association pour la Recherche sur le Cancer and Fondation de France to C.T., and from the Agence Nationale de la Recherche to F.D. (ANR06-BLAN-0211-02). L.F.M. is a Young Investigator from the Human Frontier Science Program and receives support from Fundação Luso-Americana para o Desenvolvimento and Fundação para a Ciência e a Tecnologia (PTDC/SAU-MII/69280/2006 and PTDC/SAUMII/78333/2006). We thank PICT IbiSA Imaging Facility at Curie Institute, Victor Racine and Jean-Baptiste Sibarita for providing a copy of their multidimensional image analysis program, Ilse Hurbain for help in EM quantification, Pedro Simões and Maria H. Raquel for some of the lentiviral preparations, Tanya Tolmachova for bones of Rab27 knock out mice, J. Mordoh for providing anti-CD63 FC-5.01 antibody, Rhys Allan for critical reading of the manuscript.

\section{AUTHORS CONTRIBUTION:}

MO conceived, designed, performed and analysed all experiments, and wrote the paper

NBC performed initial lentivirus screens

SK performed immunoblot, RNA analyses and cell cultures

IF performed and analysed TIRFM experiments

GR performed and analysed electron microscopy experiments and corrected the paper.

AS analysed data and corrected the paper

CFM designed the lentivirus library and prepared lentiviruses

KS analysed and quantified immunofluorescence experiments

ANH performed IP experiments

$\mathrm{PB}, \mathrm{MF}, \mathrm{RPF}, \mathrm{NH}$ contributed essential reagents

BG conceived Rab effector-related experiments

MCS contributed essential reagents, analysed data and conceived Rab effector-related experiments

CD and FD analysed TIRFM experiments and wrote the paper.

SA conceived the project, and corrected the paper

LFM conceived the screen, designed and supervised the lentivirus library production and use, analysed data and corrected the paper.

CT conceived and supervised the project, analysed data, and wrote the paper 


\section{COMPETING FINANCIAL INTERESTS}

The authors declare no competing financial interest

\section{FIGURE LEGENDS}

Figure 1: Semi-quantitative detection of exosomes in cell culture supernatants.
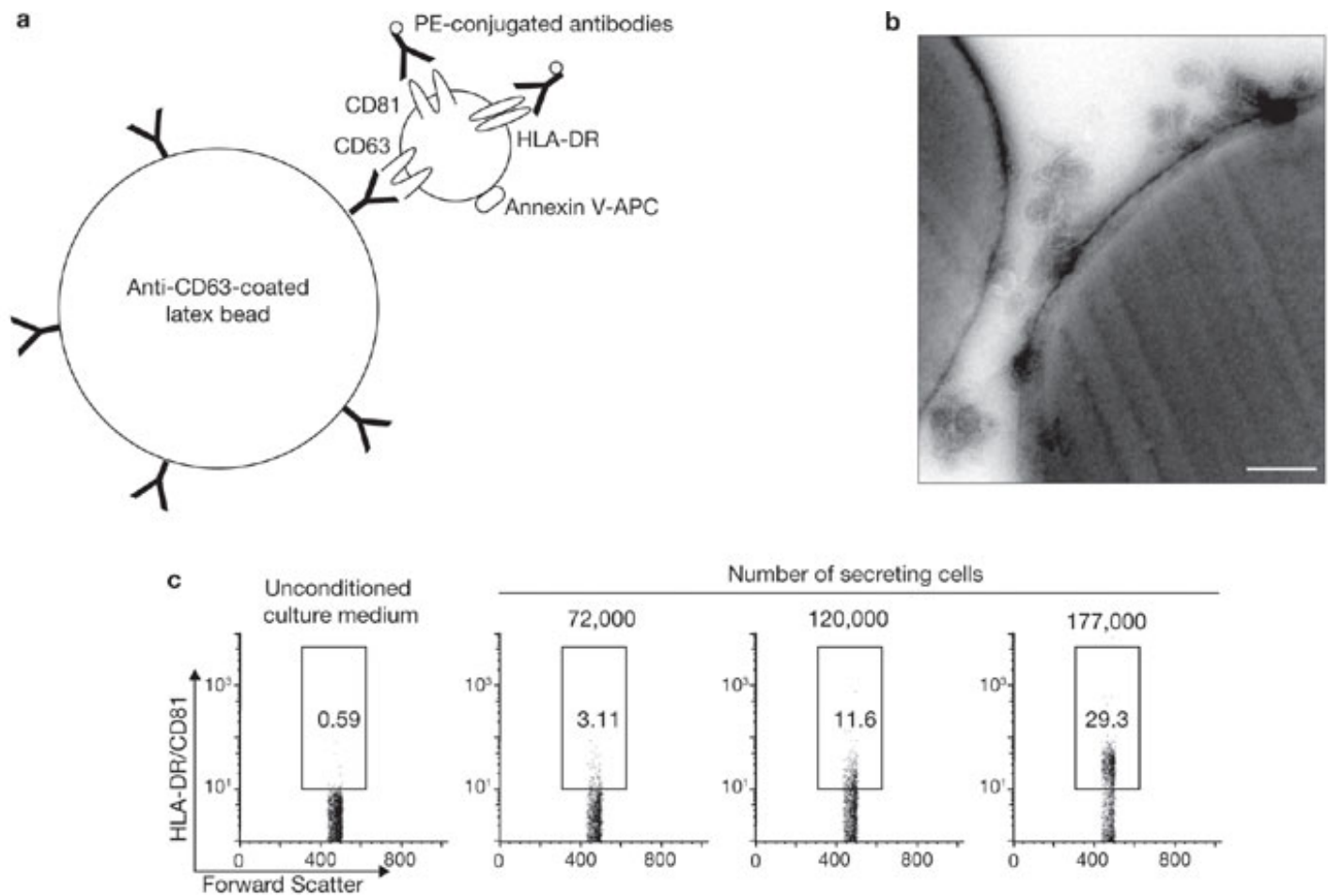

Number of secreting cells
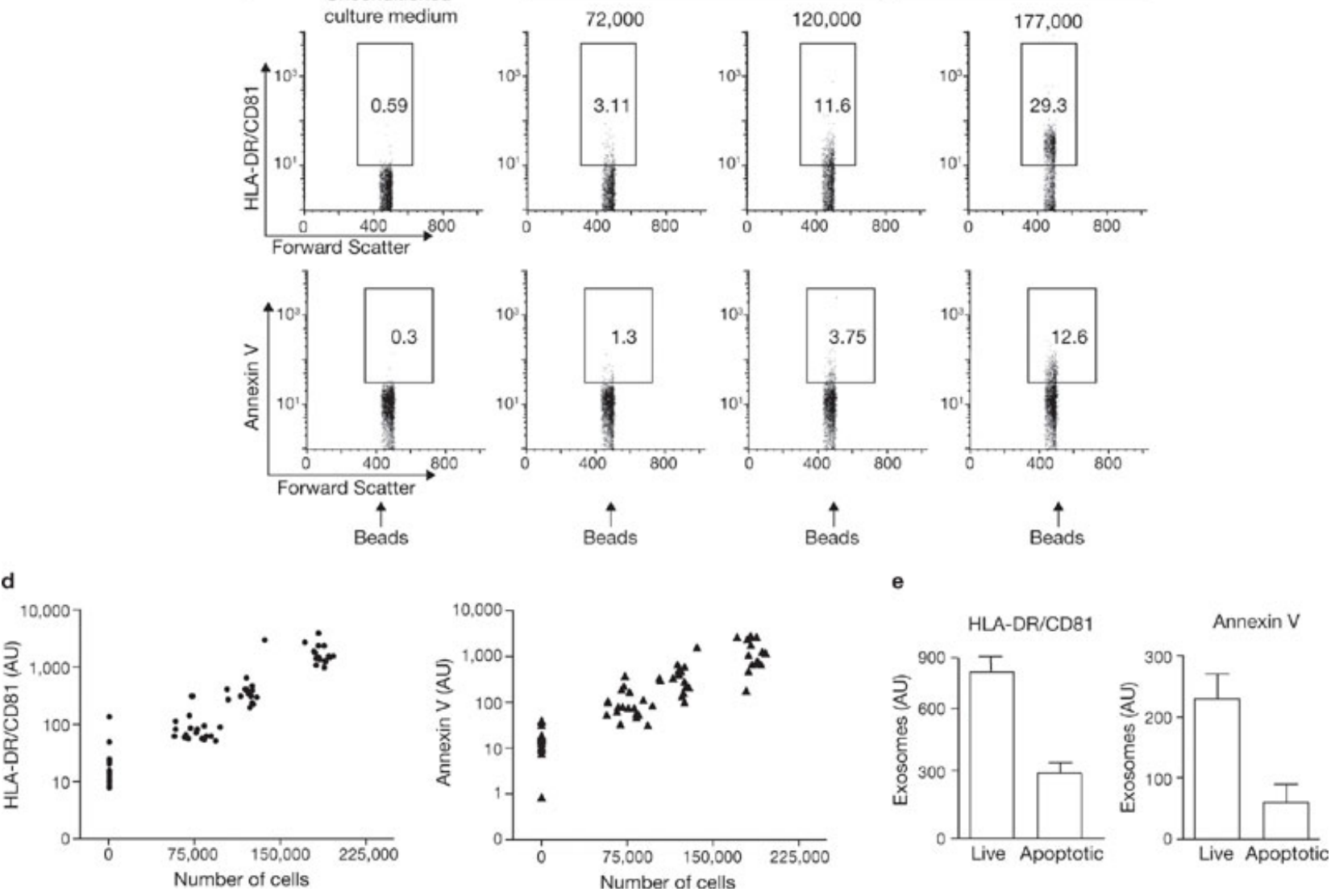

(a) Schematic drawing of the technical approach used to selectively capture and stain exosomes present in cell culture supernatants by using anti-CD63-coated beads and FACS 
detection. (b) EM image of exosomes present on the surface of anti-CD63-coated beads after incubation with supernatant from B6H4 cells. Scale bar $250 \mathrm{~nm}$. (c) Representative flow cytometry dot-plots showing HLA-DR/CD81 (top) and Annexin V (bottom) stainings of exosomes captured by beads incubated with supernatants from increasing numbers of cultured cells. Percent of positive beads are indicated inside the positive region. (d) Dose-response curve of HLA-DR/CD81 (left) and Annexin V (right) staining of exosomes captured by beads incubated with 66 different supernatants. (e) Specific detection of exosomes secreted by live cells, as compared to membranes secreted by apoptotic cells. A total of 50,000 cells either UV-irradiated or untreated were seeded in a 96-wells plate. After $12 \mathrm{~h}$ incubation, exosome depleted-medium was added and 24h later supernatants were incubated with anti-CD63coated beads and analysed by FACS. 
Figure 2: Modulation of exosome and OVA secretion by members of the Rab family.
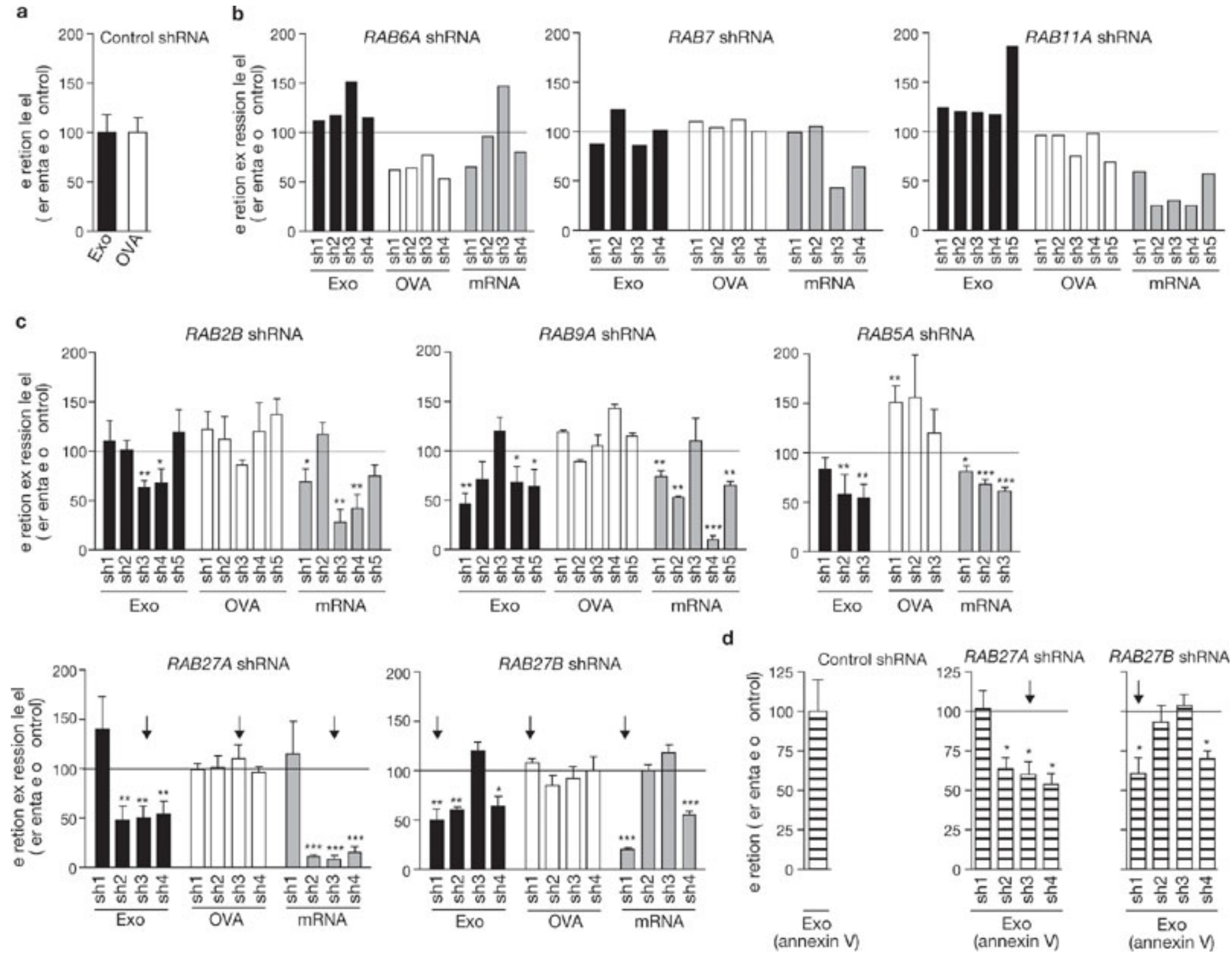
(a) Exosome secretion (black bar), measured as HLA-DR/CD81 staining and OVA secretion (white bars) by cells transduced with a control shRNA. Data are mean \pm s.d. of 3 experiments (in each experiment, $100 \%$ = mean of 3 independent wells). (b) Exosome and OVA secretion and specific mRNA levels of cells transduced with different shRNAs (each bar represents a different shRNA) targeting Rab proteins that were not selected as hits in the screen, either because neither exosome nor OVA secretion were modified (Rab7, Rab11a) or because only OVA secretion was inhibited (Rab6a). Mean values of two independent experiments are shown. (c) Exosome and OVA secretion, and specific mRNA levels in cells transduced with shRNAs targeting Rab family members that were selected as candidate hits because at least two shRNAs significantly affected exosome without affecting OVA secretion. Data are mean \pm s.d. from measures performed in supernatants from five (Rab2b, Rab9a and Rab5a) or nine 5Rab27a and Rab27b) wells present in different plates. (d) Exosome secretion by control and Rab27a- or Rab27b-KD cells was also evaluated by detection of exposed PS with Annexin V. Data are mean \pm s.d. from measures performed in supernatants from three different wells present in different plates Arrows in $\mathbf{c}$ and $\mathbf{d}$ indicate the shRNAs that were chosen for further characterization. Asterisks indicate statistically significant differences from the control. ${ }^{*} P<0.05$, ** $P<0.01$, *** $P<0.001$ (one-way ANOVA followed by Tukey's post test). Exo, exosomes. 
Figure 3: Large-scale purification and characterization of exosomes secreted by Rab27a and Rab27b-KD cells.
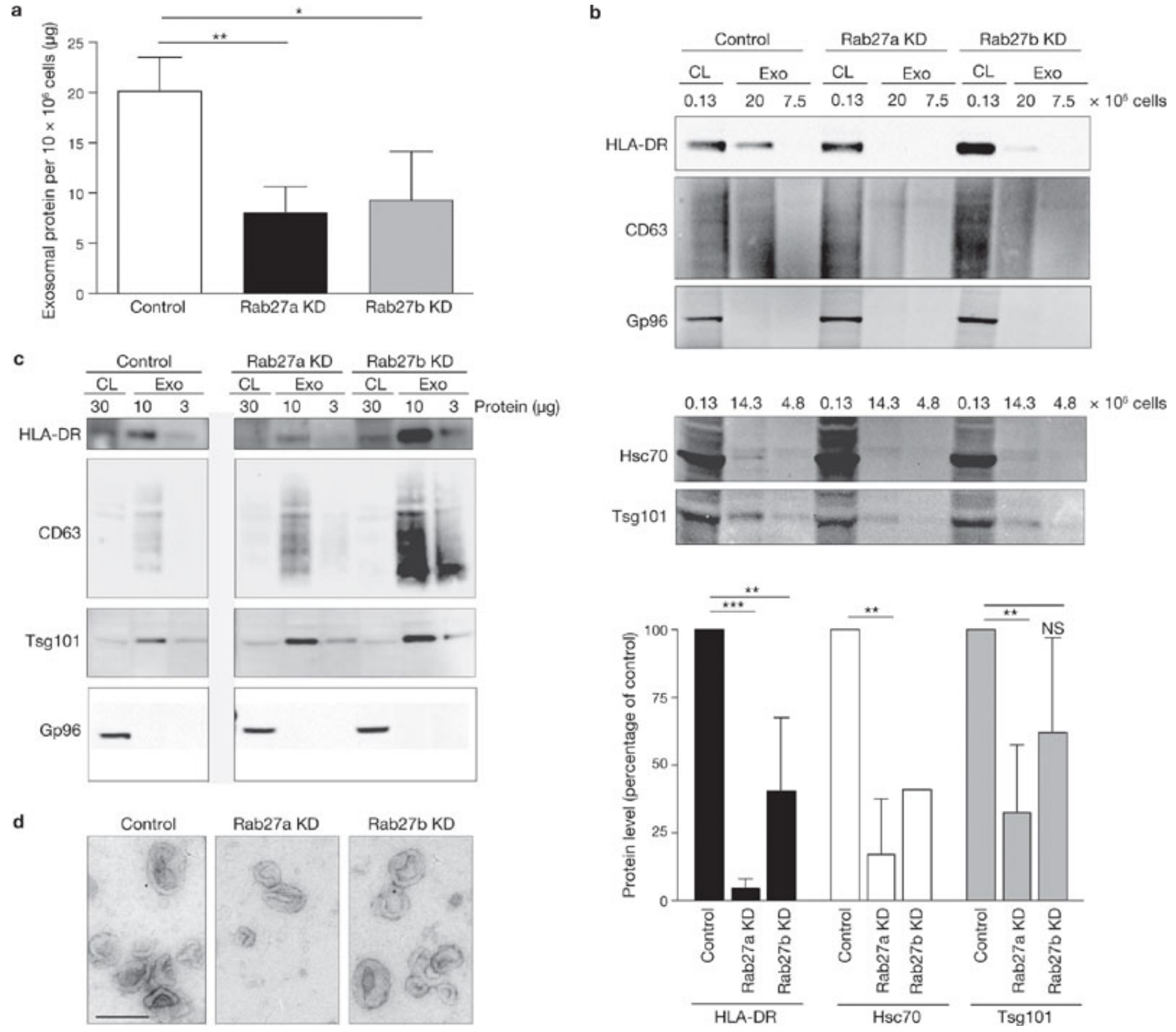

(a) The total amount of proteins in the exosomal pellet purified from large-scale cultures of stably-transduced cells was quantified by Bradford assay, and reported to the number of secreting cells. The mean \pm s.d. from 4 independent experiments are shown. (b) Characterization of exosomal proteins by immunoblotting. Purified exosomes secreted by equal amounts of control or KD cells were analysed by immunoblotting for the presence of CD63, tsg101, HLA-DR, hsc70, and gp96 proteins. A total of 130,000 cells were lysed and used for comparison (CL). One representative experiment out of four is shown. Histogram shows quantification of signal intensity of HLA-DR, hsc70 and tsg101 proteins in exosomes secreted by 20 million KD cells, normalized to the value obtained with exosomes from 
control cells (mean \pm s.d. of 3 independent experiments for HLA-DR, 4 for tsg101, 2 for hsc70). (c) Equal amounts of total exosomal proteins (quantified by Bradford assay) secreted by either control or KD cells were analysed by immunoblotting for presence of CD63, tsg101, HLA-DR, and gp96 proteins, and compared to cell lysates. (d) Exosomes purified from cell culture supernatant were negatively stained and analysed by EM. Scale bar, $200 \mathrm{~nm}$. Data were analyzed by one-way ANOVA followed by Tukey's pot test. $*=\mathrm{p}<0.05$, $* *=\mathrm{p}<0.01$, $* * *=\mathrm{p}<0.001$ 
Figure 4: Effect of Rab27a and Rab27b KD on the intracellular distribution of CD63positive compartments.
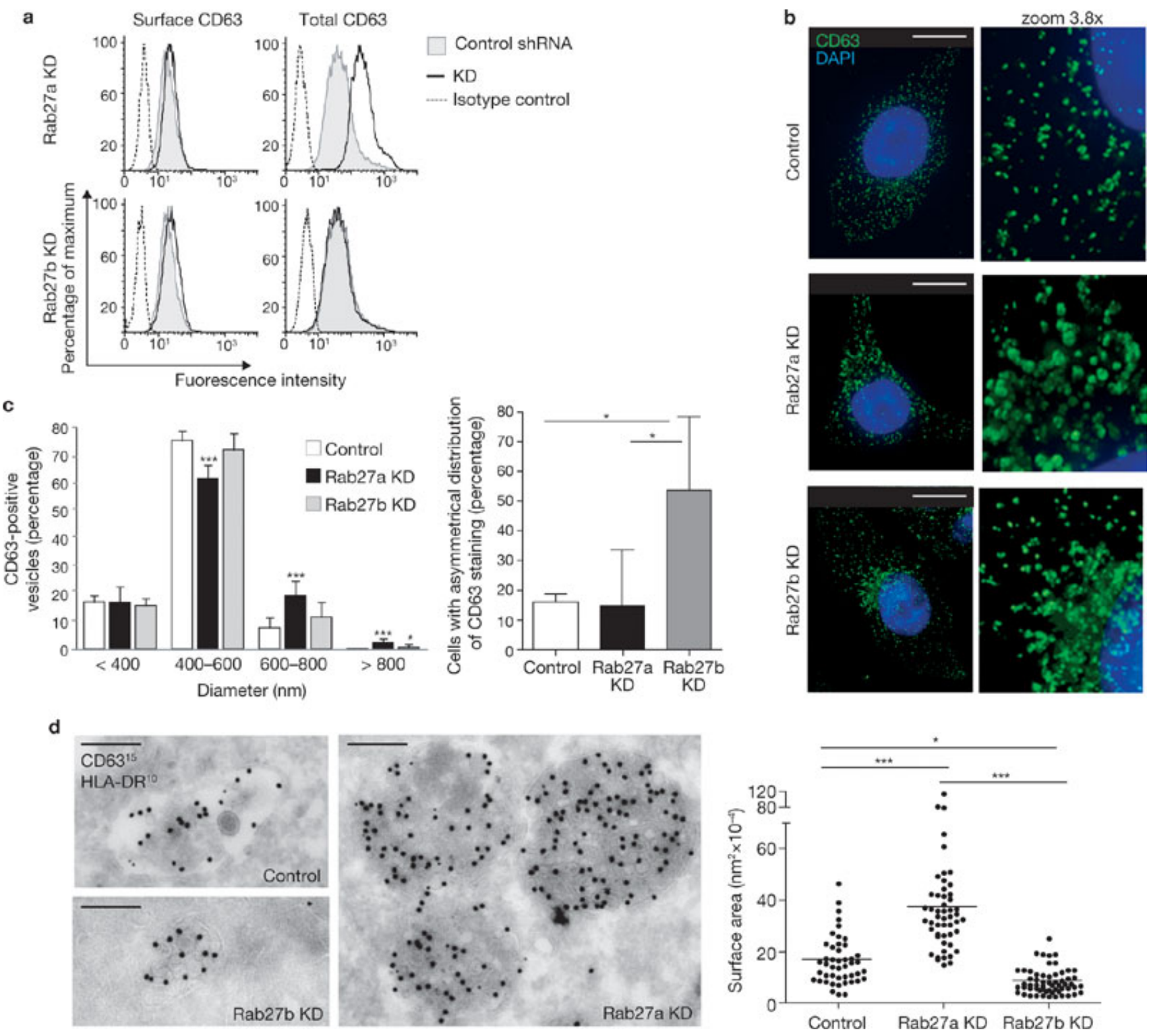

(a) Control, Rab27a- and Rab27b-knockdown cells were stained with anti-CD63 antibodies with (total) or without (surface) permeabilization, and analysed by FACS. Histograms from one representative experiment out of four are shown (dashed-line empty histograms correspond to isotype-controls). (b) Three-dimensional deconvolution fluorescence microscopy of control, Rab27a- and Rab27b-knockdown cells stained with anti-CD63 (green) and DAPI (nucleus, blue). Scale bar: $17.5 \mu \mathrm{m}$. Zooms are x 3.8. (c) Quantification of the size and distribution of CD63-positive vesicles in control (white bars), Rab27a (black bars) and Rab27b(grey bars)-knockdown cells: mean size of individual CD63-positive compartments (left ; means \pm s.d. of 9796, 8247 and 9072 vesicles counted in 14 control, 15 Rab27a- and 20 
Rab27b-knockdown cells, respectively) and percentage of cells with asymmetrical distribution (right, mean \pm s.d. of four experiments; a total of 144, 222 and 227 cells, respectively) are represented. (d) Left, electron micrographs of ultrathin cryosections of control, Rab27a-knockdown or Rab27b-knockdown cells immunogold-labelled with antiHLA-DR (10nm of gold) and anti-CD63 antibodies (15nm of gold). Scale bar, $250 \mathrm{~nm}$. Right, the surface area of individual CD63-positive multivesicular compartments was on randomly taken electron micrographs was measured. Asterisks indicate statistically significant differences. $*=P<0.05, * *=P<0.01, * * *=P<0.001$ (one-way ANOVA followed by Tukey's post test). 
Figure 5: Differential intracellular distribution of Rab27a and Rab27b.
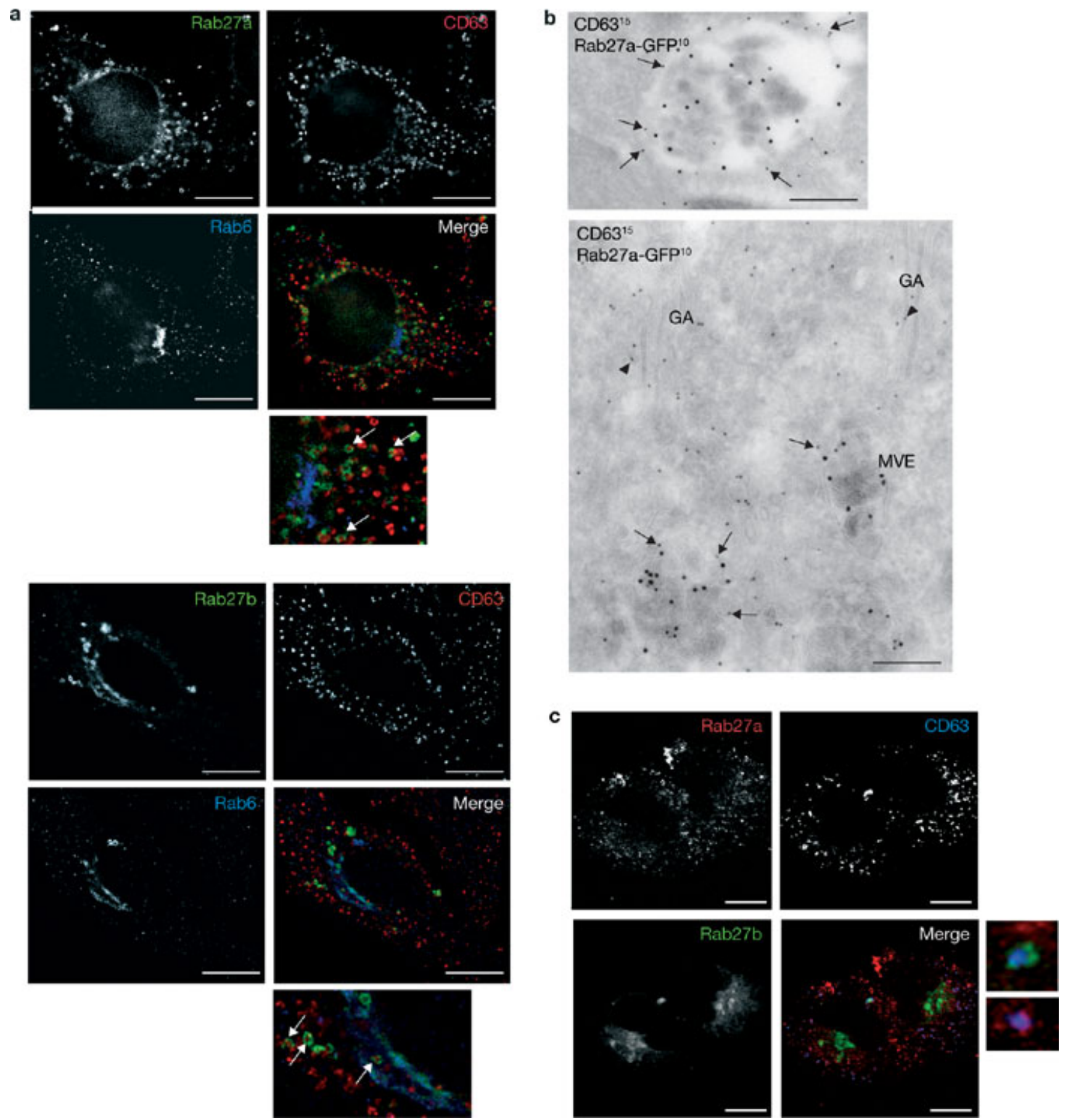

(a) 3D deconvolution fluorescence microscopy of cells transiently transfected with GFPRab27a (left panel) or GFP-Rab27b (right panel) -encoding plasmids, and subsequently stained with anti-CD63 and anti-Rab6 antibodies. Arrows point at CD63-positive compartments with GFP-Rab27 staining. Scale bar: $10 \mu \mathrm{m}$. Zoom: x 2.3. (b) Electron micrographs of ultrathin cryosections of GFP-Rab27a- (left panel) or GFP-Rab27b- (right panel) transfected cells, immunogold-labeled with anti-CD63 (15 nm gold) and anti-GFP 
(10nm gold) antibodies. Bar, $500 \mathrm{~nm}$. Arrows indicate Rab27 association with the limiting membrane of MVEs and arrowheads indicate the localization of Rab27b in the Golgi apparatus area. (c) Confocal fluorescence microscopy of cells doubly transfected with mCherry-Rab27a- and GFP-Rab27b-encoding plasmids, and subsequently stained with antiCD63. Scale bar: $10 \mu \mathrm{m}$. Zoom: x 8. 
Figure 6: Effect of Rab27a and Rab27b KD on the mobility of CD63-positive compartments in the subplasmalemmal region analysed by TIRF microscopy.

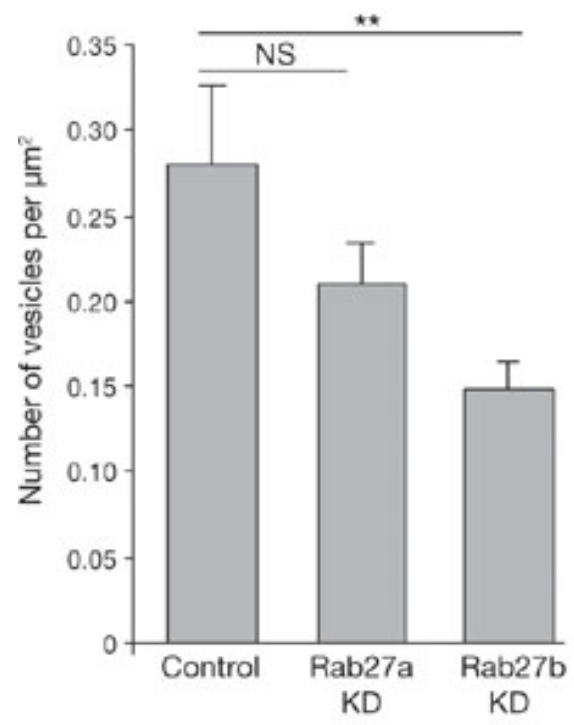

c
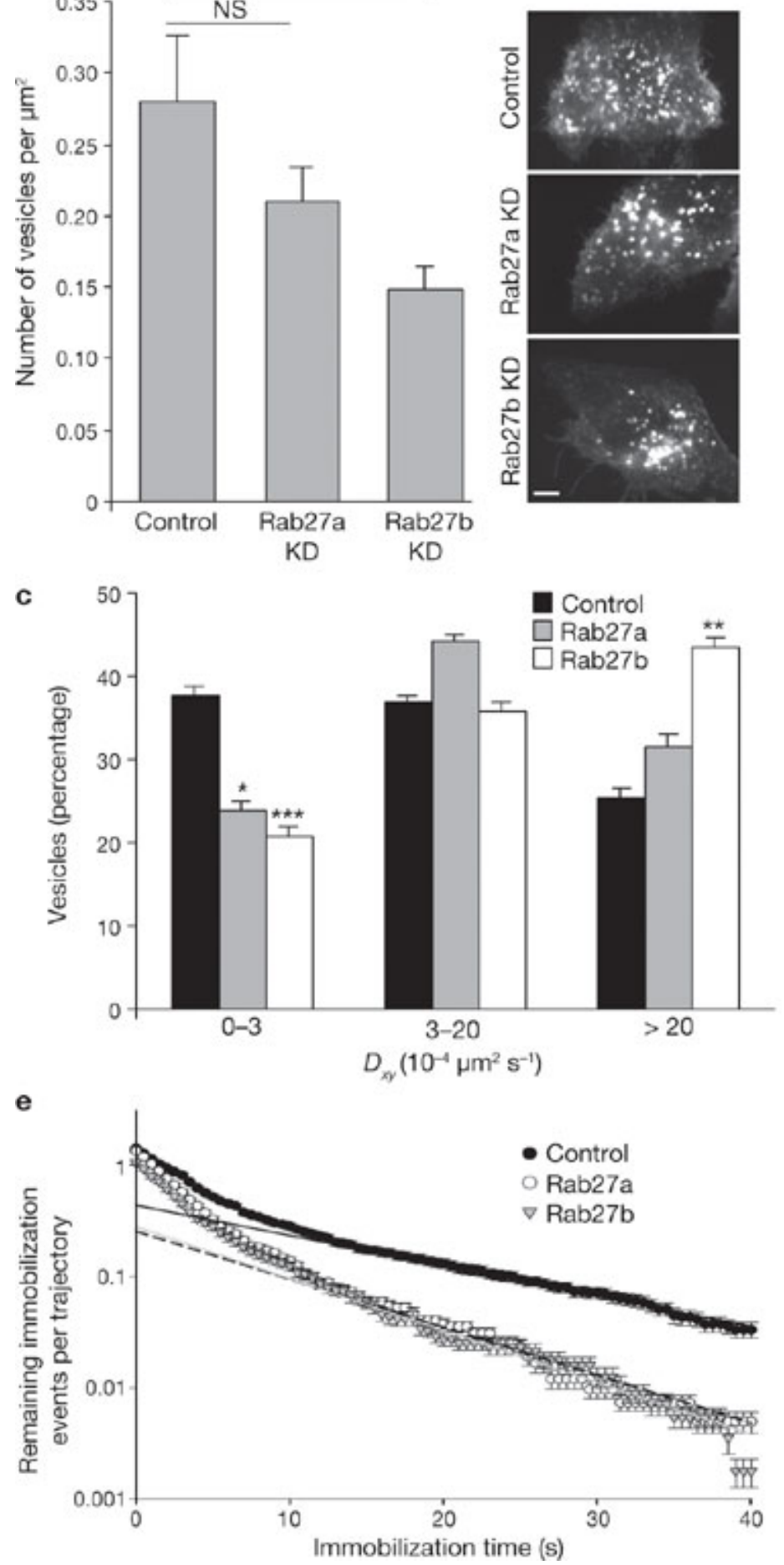

b

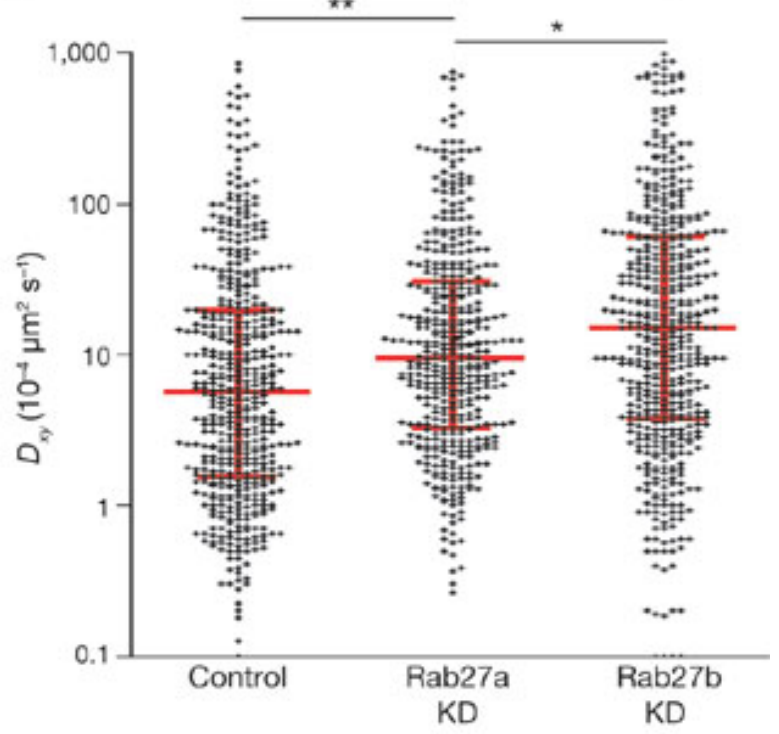

d $507 \begin{aligned} & \square \text { Always immobile } \\ & \text { QNever immobile }\end{aligned}$

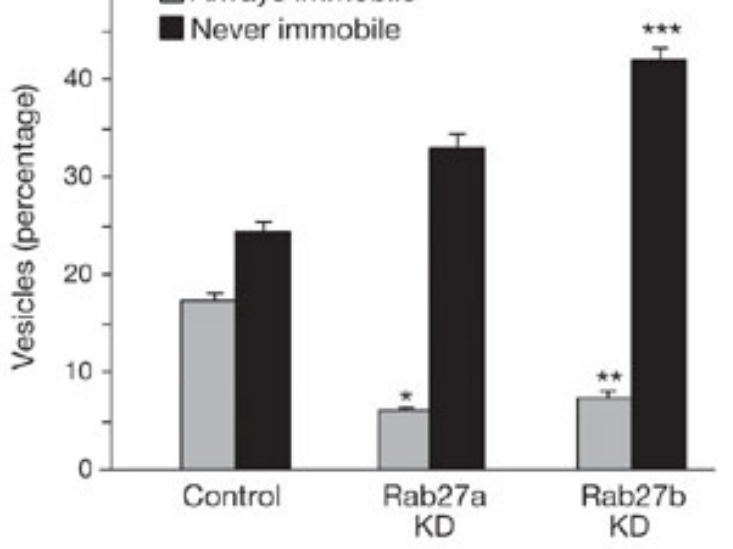

f

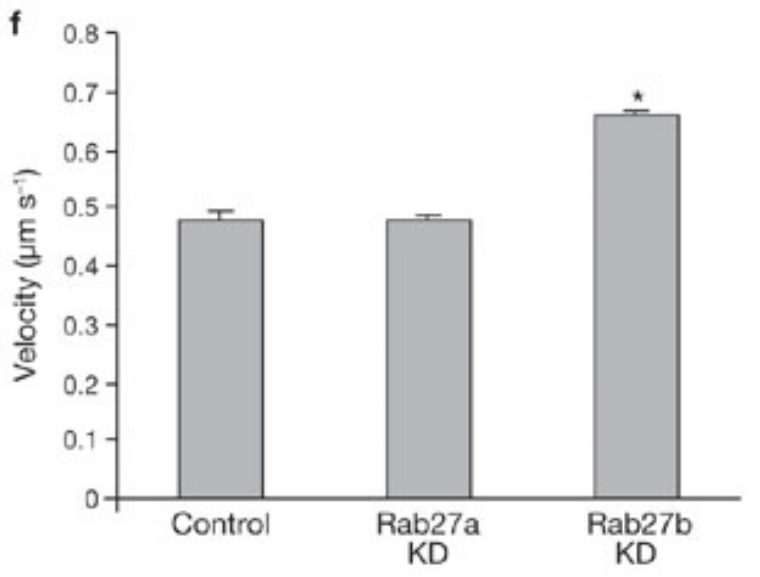

(a) Right, representative TIRFM images showing GFP-CD63 distribution in control, Rab27aand Rab27b-knockdown cells. Scale bar: $5 \mu \mathrm{m}$. Left, vesicle density in the subplasmalemmal region detected by TIRFM (data are the mean \pm s.e of vesicles quantified in 18 control, 19 
Rab27a- and 20 Rab27b-knockdown cells, Mann-Whitney, **,P= 0,0042). (b) Effect of Rab27a and Rab27b knockdown cells on vesicle motion. Mean diffusion coefficient $\left(D_{x y}\right)$ values of individual trajectories obtained for at least 400 vesicles in control, Rab27a- and Rab27b-knockdown cells are shown. Red bars: median and interquartile ranges $(* *=p<0.01$, $* * *=\mathrm{p}<0.001$, Kruskal Wallis test, followed by Dunn's post test,). (c-f) Entire trajectories were analyzed using a rolling analysis window: black bars, control: 12 cells, 499 trajectories; grey bars, Rab27a knockdown: 10 cells, 415 trajectories; white bars, Rab27b knockdown: 12 cells, 528 trajectories. (c) Data show the mean \pm s.e.m of $D_{x y}$ values computed on entire trajectories. Rab27a and Rab27b silencing reduced the percentage of vesicles with $D_{x y}<3 \mathrm{x}$ $10^{-4} \mathrm{\mu m}^{2} / \mathrm{s}$ that are presumably docked $(* P=0.0479$, $* * P=0.0079)$. (d) Data show the percentage of vesicles that were always (grey bars) or never (black bars) docked ( $D_{x y}<3 \mathrm{x}$ $10^{-4} \mu \mathrm{m}^{2} / \mathrm{s}$ ) in control, Rab27a- and Rab27b-knockdown cells. ${ }^{*} P=0.0147$, $* * P=0.0086$. (e) Further characterization of docking events. Immobilization periods were detected as in (d) and their duration was measured. Logarithmic plots of survival curves for immobilization times are shown (control, black line; Rab27a knockdown, dashed line; Rab27b knockdown, gray line). The best fit was obtained with the sum of two exponentials $\left(r^{2}>0.998\right)$ and was significantly different in Rab27 knockdown cells compared to control cells $(p<0.0001$; extra sum-of-squares F-test). (f) Rab27b knockdown increases mobility along microtubules. The mean \pm s.e.m velocity of vesicles during periods of directed motion is shown $\left({ }^{*} P=0.011\right)$. 
Figure 7: Effect of Rab27 effector proteins on exosome secretion.
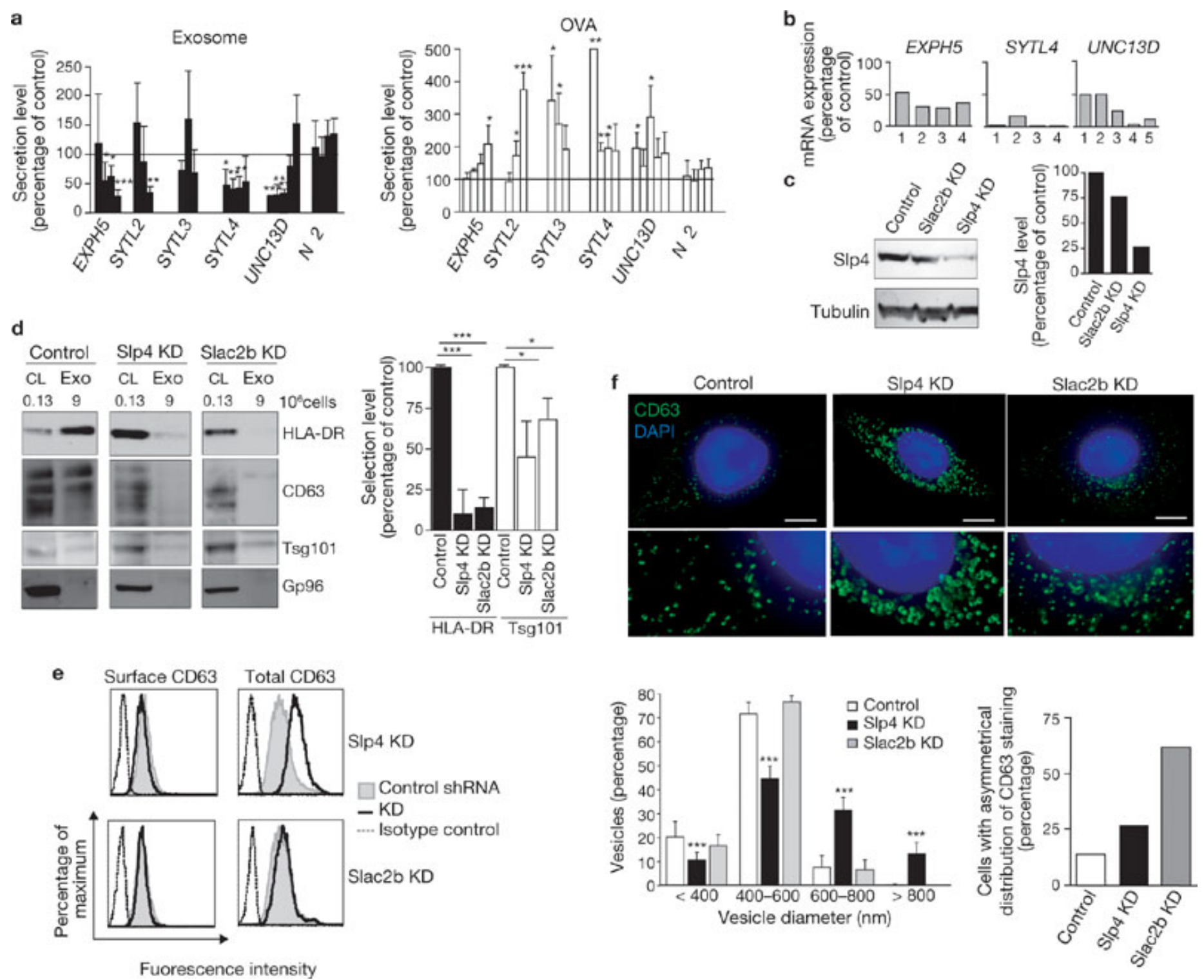
(a) FACS analysis of exosome (HLA-DR/CD81 staining, black bars) and OVA (white bars) secretion by cells transduced with shRNA to Rab27 effector proteins (3 to 5 viruses/gene). Mean \pm s.d. of three independent experiments is shown. (b) Specific inhibition of Slp4, Slac2b and Munc13-4 gene expression, as assessed by qRT-PCR. Results from 2 experiments performed in triplicates, expressed as percent of mRNA expression of control cells are shown. (c) Immunoblot (left panel) and quantification (right panel) of Slp4 protein level in Slp4 and Slac2b KD cells. Tubulin was used as a loading control. (d) Exosome secretion by B6H4 cells stably transduced with scrambled shRNA (Control), Slp4 shRNA \#3, or Slac2b shRNA \#3. Exosomes secreted by 9 million cells (Exo), or $30 \mu \mathrm{g}$ of total cell lysates (CL) were analysed by immunoblotting for presence of CD63, tsg101, HLA-DR and gp96 proteins. One representative experiment of three is shown. The relative amount of HLA-DR and tsg101 secreted in exosomes was quantified by signal intensity measurement (right panel). Results are expressed as the mean $\pm \mathrm{SD}$ of ratio to control cells in 3 independent experiments. (e) FACS analysis of Slp4 KD, Slac2b KD and control cells stained in parallel with anti-CD63 antibodies after permeabilization (total) or not (surface). Representative histograms (left panels, thin-lined empty histograms correspond to isotype-controls) of three independent experiments are shown. (f) Three-dimensional deconvolution fluorescence microscopy of control, Slp4-, and Slac2b knockdown cells stained with anti-CD63 (green) and DAPI (nucleus, blue). Upper panels, representative images of control, Slp4- and Slac2b-knockdown cells. Scale bars, $10 \mu \mathrm{m}$. Zooms are x 2.5. Lower panels: quantification of CD63-positive vesicles size (left panel, mean \pm SD of 10106, 11430 and 11404 vesicles from 20 control, 21 Slp4- and 23 Slac2b-knockdown cells, respectively) and cells with asymmetrical distribution of CD63-positive vesicles (right panel, mean \pm SD of 2 independent experiments, a total of 107 control, 86 Slp4- and 115 Slac2b-knockdown cells). $*=\mathrm{p}<0.05$, **= $\mathrm{p}<0.01$, ***= $\mathrm{p}<0.001$ (one-way ANOVA followed by Tukey's post test). KD, knockdown. For full scans of blots in c and d, see Supplementary information, Fig.S6. 
Figure 8: Interaction between Slp4 and Rab27a.

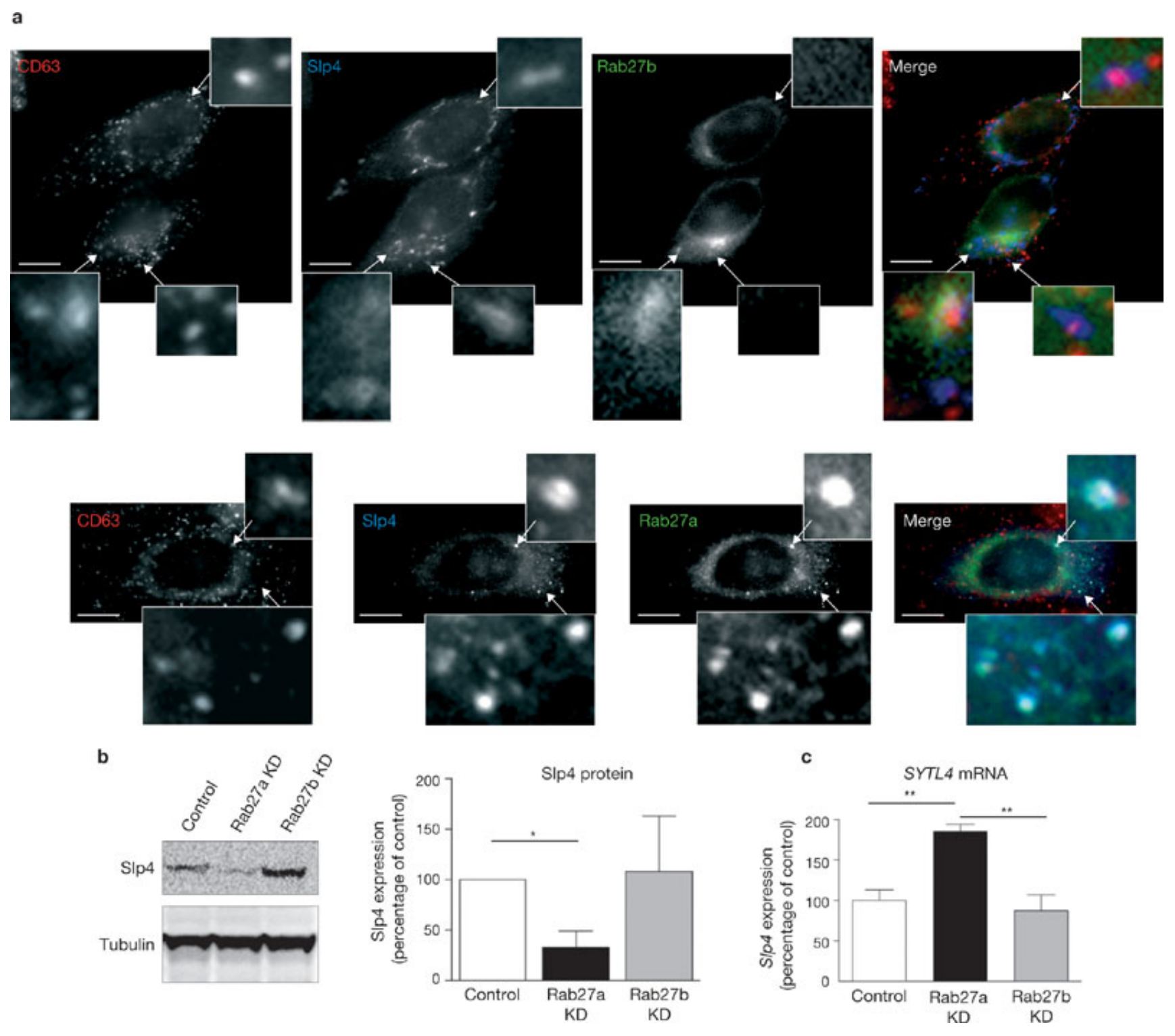


(a) 3D deconvolution fluorescence microscopy of cells doubly transfected with T7-Slp4 and either GFP-Rab27b-(top panel) or GFP-Rab27a-(bottom panel) encoding plasmids, and subsequently stained with anti-CD63 and anti-Slp4 antibodies. Arrows point at zoomed areas (x8). (b-c) Slp4 expression levels in cells KD for either Rab27a or Rab27b. Slp4 protein levels were evaluated by immunoblotting in cells KD for either Rab27a or Rab27b (b). A representative blot (left panel) and the quantification of the relative mean intensity \pm s.d. of three independent blots normalized to tubulin levels (right panel) are shown. Uncropped western blots are shown in Fig. S8b. (c) The level of expression of Slp4-specific mRNA in cells KD for either Rab27a or Rab27b was analysed by qPCR and normalized to the level of expression of GAPDH. Mean values \pm s.d. of three independent experiments are shown. Data were analyzed byone-way ANOVA followed by Tukey's pot test. Stars indicate statistically significant differences with control cells. $*=\mathrm{p}<0.05$, $* *=\mathrm{p}<0.01, * * *=\mathrm{p}<0.001$

\section{METHODS}

\section{Cells and reagents}

HeLa-CIITA cells ${ }^{42}$ were stably transfected with pcDNA3 encoding the OVA cDNA fused to the signal peptide of MFG-E8/lactadherin ${ }^{13}$, and a subclone named B6H4 was selected. These cells were cultured in DMEM containing 4.5 g glucose/l (Invitrogen) 10\% fetal calf serum (Abcys), $2 \mathrm{mM}$ glutamine, $100 \mathrm{IU} / \mathrm{ml}$ penicillin, $100 \mu \mathrm{g} / \mathrm{ml}$ streptomycin, $300 \mu \mathrm{g} / \mathrm{ml}$ hygromycin B (Calbiochem), $500 \mu \mathrm{g} / \mathrm{ml}$ geneticin (Invitrogen).

Plasmids used for transient transfections (performed with Bio-Rad gene pulser $\mathrm{II}^{42}$ ) include pEGFPC3-Rab27a $^{43}$, pEGFPC3-Rab27b ${ }^{23}$, pEGFP-bos-CD63 ${ }^{44}$, pT7-Slp4 ${ }^{45}$, mCherryRab27a generated by PCR from pcDNA3.1mcherry ${ }^{46}$ and pEGFPC3-Rab27a, and cloned into pCMVMyc vector $^{38}$ (A.H. Hume, unpublished).

Lentiviruses expressing shRNA were obtained from the RNAi consortium (TRC) and produced as previously described ${ }^{47}$. Sequences of the shRNA used for large scale cultures are: Rab27a: GCTGCCAATGGGACAAACATA, Rab27b : CCCAAATTCATCACTACAGTA, Slp4 : CCTCCCTTTACATGGAAAGAT; Slac2b : CCCAAACTAGAAGAAGTTCTT.

Antibodies for FACS analysis: mouse anti-human CD63 (FC-5.01, Zymed) and goat antiOVA (ICN) for coupling to beads, PE-conjugated anti-HLA-DR (L243, BD) and anti-CD81 (BD), rabbit anti-OVA (Sigma) coupled to Alexa488 according to manufacturer instruction 
(Pierce). For immunoblotting: goat anti-tsg101 (Santa Cruz), rat anti-gp96 and -hsc70 (Stressgen), mouse anti-CD63 (Zymed), -HLA-DR (clone 1B5), and -Rab27a (clone 4B12 ${ }^{43}$ ), rabbit anti-Rab27b ${ }^{35}$, $-S l p 4{ }^{33}$, and $-S l a c 2 b^{33}$, and HRP-conjugated secondary antibodies (Jackson Immunoresearch). For immunofluorescence and immunoEM: mouse anti-CD63 (Zymed), rabbit anti-Rab6 (Santa Cruz) and -GFP (Invitrogen), Alexa488-anti-mouse and Cy3-anti-rabbit (Jackson Immunoresearch), DAPI (Molecular Probes).

\section{Immunoadsorbtion and detection of exosomes by FACS}

Anti-human CD63 or anti-OVA antibodies were coupled to $4 \mu \mathrm{m}$ aldehyde-sulfate beads (Invitrogen) by incubating $35 \mu \mathrm{g}$ of antibody with $1 \times 10^{8}$ beads followed by blocking of remaining activated groups with PBS-4\% BSA.

Cell culture supernatants were cleared by centrifugations at 1,200 rpm (for $5 \mathrm{~min}$ ) and 4,000 rpm (for $20 \mathrm{~min}$ ). Cleared supernatants (100 $\mu \mathrm{l}$ ) were incubated with 20,000 anti-CD63 and 20,000 anti-OVA coupled beads overnight at room temperature with 6,500 rpm shaking. Beads were washed twice in PBS-2\% BSA and incubated with PE-anti-HLA-DR + PE-antiCD81 and Alexa488-anti-OVA (all at 1:100 dilution) for 30 min on ice. Beads were washed twice in annexin V buffer (BD Biosciences) and incubated with APC-conjugated annexin V (1:10) for 10 minutes. Beads were acquired on a FACSCalibur (BD) and data were analysed with FlowJo software (Tree Star). Threshold of negative staining was obtained with beads incubated with unconditioned medium, and for each culture condition, arbitrary units (AU) of exosome or OVA secretion were calculated as a percentage of positive beads $\mathrm{x}$ MFI of positive beads. To rule out detection of apoptotic blebs, a total of 50,000 cells either UVirradiated or untreated were seeded in a 96-wells plate. After $12 \mathrm{~h}$ incubation, exosome depleted-medium was added and 24h later supernatants were incubated with anti-CD63coated beads and analysed by FACS.

\section{Lentiviral infection and screening procedure}

HeLa B6H4 cells were plated in 96 well round-bottom plates at 2,500 cells per well (day zero). At day 1 , cells were infected with lentiviral vectors in the presence of $8 \mu \mathrm{g} / \mathrm{ml}$ polybrene and $5 \mu \mathrm{g} / \mathrm{ml}$ puromycin was added at day 2. Medium was replaced with exosomedepleted medium at day 4. Supernatants were collected $48 \mathrm{~h}$ later for OVA and exosome quantification, and the amount of live cells in each well was determined using Cell titer blue 
reagent viability assay (Promega). OVA and exosome secretions per well were normalized to the number of viable cells, and only wells containing at least 75,000 cells were analysed. In each experiment, the basal level of exosome or OVA secretion (= control) was calculated as either A.U. of cells transduced with control scrambled shRNA, or mean of A.U. of one entire plate. A candidate gene was defined as a hit if two or more shRNAs modified A. U. of exosome secretion, in duplicate, by more than one standard deviation from the control. Results are presented as percent of A.U. of control.

\section{Quantitative RT-PCR}

RNAs were isolated from each well at day 5 after lentiviral infection with the Qiagen RNeasy Mini Kit and 200 ng were reverse transcribed with AMV RT (Finnzymes). 1/10th of cDNA was used for each PCR reaction, performed with SYBRgreen (Thermo Scientific) on ABI PRISM 7900 real-time thermal cycler (Applied Biosystems). Primer sequences for each gene were either retrieved from Primer Bank (http://pga.mgh.harvard.edu/primerbank/) or designed using Primer3 (http://frodo.wi.mit.edu/cgi-bin/primer3/primer3_www.cgi). Cycle thresholds (Ct) were normalized to Ct of GAPDH and fold enrichments were calculated as compared to the control shRNA-transduced cells values.

\section{Exosome purification}

Cells were cultured in "exosome-depleted medium" (complete medium depleted of FCSderived exosomes by overnight centrifugation at 100,000 g) for 48 hours and exosomes were purified by filtration on $0.22-\mu \mathrm{m}$ pore filters, followed by ultracentrifugation at $100,000 \mathrm{~g}$ as described $^{24}$. In each exosome preparation, the concentration of total proteins was quantified by Bradford assay (BioRad).

\section{Electron microscopy}

Exosomes fixed in 2\% paraformaldehyde were loaded on Formwar/carbon-coated EM grids. Samples were post-fixed in 1\% glutaraldehyde, contrasted and embedded as previously described $^{5}$. For immunolabeling, cells were fixed with $2 \%$ paraformaldehyde in $0.2 \mathrm{M}$ phosphate buffer $\mathrm{pH} 7.4$ and processed for ultrathin sectioning and immunolabeling with the indicated antibodies and protein A coupled to 10 or $15 \mathrm{~nm}$ gold particles (Cell Microscopy 
Center, The Netherlands) as described previously ${ }^{5}$. Sections were observed at $80 \mathrm{kV}$ with a CM120 Twin Philipps electron microscope (FEI Company, The Netherlands) and digital acquisitions were made with a numeric camera (Keen View; Soft Imaging system, Germany). Quantification of the surface of MVBs was performed on at least 40 CD63-positive multivesicular compartments present in randomly taken pictures analysed using the iTEM software (Olympus).

\section{Immunoblotting}

Cells were lysed in $50 \mathrm{mM}$ Tris, pH 7.5, 0.3 M NaCl, 0.5\% Triton X-100, 0.1\% sodium azide, with a cocktail of antiproteases (Roche), and cleared from nuclei by centrifugation at 13,000 rpm for $5 \mathrm{~min}$. Protein extracts were separated on 4\%-12\% SDS-PAGE, blotted on Immobilon (Millipore), and HRP-conjugated secondary antibodies were revealed using chemoluminescence substrate (Roche). Images were acquired using Chemidoc XRS (BioRad), intensity of the bands was quantified using QuantityOne software (Biorad) and results were expressed as the ratio with control cells.

\section{Flow Cytometry}

For surface labeling, cells were stained with antibodies diluted in PBS-0.5\% BSA on ice. For intracellular + surface staining, cells fixed with $1 \%$ paraformaldehyde, were quenched with PBS-0.1M glycine, permeabilized using PermWash reagent (BD), and stained with antibodies diluted in permeabilization buffer. Cells were acquired on a FACScalibur and analysed using FlowJo software.

\section{Immunofluorescence Microscopy}

Cells $\left(1 \times 10^{5}\right)$ were seeded on glass coverslips for $18 \mathrm{~h}$, fixed in $4 \%$ paraformaldehyde, quenched with $0.1 \mathrm{M}$ glycine, permeabilized in PBS-0.2\% BSA-0.05\% saponin, and incubated with primary and secondary fluorescent antibodies. The coverslips mounted with Fluoromount-G (Southern Biotechnology Associates) were examined under a motorized upright wide-field microscope (Leica DMRA2) equipped for image deconvolution. Acquisition was performed using a x100 objective (PL APO HCX, 1.4 NA) and a highsensitive cooled interlined CCD camera (Roper CoolSnap HQ). Z-positioning was 
accomplished by a piezo-electric motor (LVDT, Physik Instrument) and Z-series of images were taken every $0.2 \mu \mathrm{m}$. Images were acquired with Metamorph Software. Vesicle sizes were calculated with the Multidimensional Image Analysis (MIA) interface based on wavelet decomposition $^{48}$, running under MetaMorph (Universal Imaging Corporation). Briefly, fluorescent objects corresponding to MVE were detected as fluctuations that are 20-fold larger than the noise. The watershed function was routinely applied in order to precisely detect individual structures in dense regions. The diameter of each structure was calculated from the sum of pixels assuming a spherical shape. Alternatively, images were acquired with Zeiss confocal microscope (LSM Axivert 720) using $63 \times 1.4$ NA oil immersion objective. Image acquisition and Image analysis were performed in the PICT IbiSA Imaging Facility (Curie Institute). Brightness and contrast of the images displayed in Figures 4b, 5a, $7 \mathrm{f}$ and 8a were adjusted for better visualisation according to the journal's guidelines for presentation of digital data.

Quantification of asymmetrical distribution of CD63-positive vesicles was performed manually in a double blind setting. Asymmetry was defined as a concentration of the CD63staining in one side of the nucleus (covering less than $50 \%$ of the nucleus diameter).

\section{Evanescent wave fluorescence microscopy (TIRFM)}

B6H4 cells stably expressing shRNA were transfected with GFP-CD63-encoding plasmid 36h before analysis by TIRFM. TIRF imaging was performed on a custom setup as described previously $^{26}$. The penetration depth $\delta$ of the evanescent field used to excite the fluorophore was set to $150 \mathrm{~nm}$. Frames were acquired at $10 \mathrm{~Hz}$ in stacks of 450 images with an exposure time of $100 \mathrm{~ms}$. One pixel corresponds to $107.5 \mathrm{~nm}$. The density of GFP-CD63 structures was evaluated using $\mathrm{MIA}^{48}$. The quantification was performed on regions devoid of TGN-like labeling and expressed as the number of observed CD63-positive structures divided by the size of the cell footprint. From stacks of images, 2-dimensional (x, y) trajectories were obtained by single particle tracking using MIA software. Mean square displacement (MSD) in the $\mathrm{x}, \mathrm{y}$ plane was calculated as described ${ }^{25,}{ }^{49}$. The diffusion coefficient $D_{x y}$ was then calculated as $D_{x y}=s / 4$ with $s$ being the slope of the linear fit of the first 15 points of the MSD curve. To measure $D_{x y}$ variations along a given trajectory, a rolling analysis window of $2.5 \mathrm{~s}$ was used as described ${ }^{25}$. Using this method, a $D_{x y}$ value was attributed to each point of the trajectory. Immobilization periods correspond to portions of trajectories during which $D_{x y}<3$ $\mathrm{x} 10^{-4} \mathrm{\mu m}^{2} . \mathrm{s}^{-1}$. Survival curves for immobilization times (i.e. the number of events/trajectory 
lasting at least the indicated immobilization time) were computed for each cell and averaged over the different cells. The best fit for the curves was obtained with the sum of 2 exponentials, $f N(t)=N_{1} \exp ^{(-\mathrm{t} / \tau 1)}+N_{2} \exp ^{(-\mathrm{t} / \tau 2)}$, using SigmaPlot (Systat Software, USA). $N_{1}$ and $N_{2}$ represent the abundance of each component, $\tau_{1}$ and $\tau_{2}$ their time constants. Portions of trajectories displaying directed motion were detected by inspection of $x v s t$ and $y$ vs $t$ plots and selected using locally written routines (MatLab, The MathWorks, USA). Frame-to-frame rates of movement were calculated and averaged over each period of directed motion.

\section{Statistical analyses}

Data were analysed using GraphPad Prism (GraphPad Software). Normality of the data was tested using the Kolmogorov-Smirnov test. Based on the normality test, either one-way ANOVA followed by Tukey's post test or Kruskal Wallis followed by Dunn's post test were used for multiple comparison analyses. Values are given as mean \pm S.D. in all figures except Fig.6 : mean \pm S.E.

\section{REFERENCES}

1. Thery, C., Zitvogel, L. \& Amigorena, S. Exosomes: composition, biogenesis and function. Nat Rev Immunol 2, 569-579 (2002).

2. Fevrier, B. \& Raposo, G. Exosomes: endosomal-derived vesicles shipping extracellular messages. Curr Opin Cell Biol 16, 415-421 (2004).

3. Johnstone, R.M. Exosomes biological significance: A concise review. Blood Cells Mol Dis 36, 315-321 (2006).

4. Lakkaraju, A. \& Rodriguez-Boulan, E. Itinerant exosomes: emerging roles in cell and tissue polarity. Trends Cell Biol 18, 199-209 (2008).

5. Raposo, G. et al. B lymphocytes secrete antigen-presenting vesicles. J Exp Med 183, 1161-1172 (1996).

6. Zitvogel, L. et al. Eradication of established murine tumors using a novel cell-free vaccine: dendritic cell-derived exosomes. Nat Med 4, 594-600 (1998).

7. Thery, C. et al. Indirect activation of naive CD4+ T cells by dendritic cell-derived exosomes. Nat Immunol 3, 1156-1162 (2002).

8. Wiley, R.D. \& Gummuluru, S. Immature dendritic cell-derived exosomes can mediate HIV-1 trans infection. Proc Natl Acad Sci U S A 103, 738-743 (2006).

9. Fevrier, B. et al. Cells release prions in association with exosomes. Proc Natl Acad Sci U S A 101, 9683-9688 (2004).

10. Rajendran, L. et al. Alzheimer's disease beta-amyloid peptides are released in association with exosomes. Proc Natl Acad Sci U S A 103, 11172-11177 (2006).

11. Wolfers, J. et al. Tumor-derived exosomes are a source of shared tumor rejection antigens for CTL cross-priming. Nat Med 7, 297-303 (2001).

12. Iero, M. et al. Tumour-released exosomes and their implications in cancer immunity. Cell Death Differ 15, 80-88 (2008). 
13. Zeelenberg, I.S. et al. Targeting tumor antigens to secreted membrane vesicles in vivo induces efficient antitumor immune responses. Cancer Res 68, 1228-1235 (2008).

14. Futter, C.E., Collinson, L.M., Backer, J.M. \& Hopkins, C.R. Human VPS34 is required for internal vesicle formation within multivesicular endosomes. $J$ Cell Biol 155, 1251-1264 (2001).

15. Pan, B.T., Teng, K., Wu, C., Adam, M. \& Johnstone, R.M. Electron microscopic evidence for externalization of the transferrin receptor in vesicular form in sheep reticulocytes. J Cell Biol 101, 942-948 (1985).

16. Booth, A.M. et al. Exosomes and HIV Gag bud from endosome-like domains of the T cell plasma membrane. J Cell Biol 172, 923-935 (2006).

17. Zerial, M. \& McBride, H. Rab proteins as membrane organizers. Nat Rev Mol Cell Biol 2, 107-117 (2001).

18. Seabra, M.C., Mules, E.H. \& Hume, A.N. Rab GTPases, intracellular traffic and disease. Trends Mol Med 8, 23-30 (2002).

19. Ali, B.R. \& Seabra, M.C. Targeting of Rab GTPases to cellular membranes. Biochem Soc Trans 33, 652-656 (2005).

20. Escola, J.M. et al. Selective enrichment of tetraspan proteins on the internal vesicles of multivesicular endosomes and on exosomes secreted by human B-lymphocytes. $J$ Biol Chem 273, 20121-20127 (1998).

21. Morelli, A.E. et al. Endocytosis, intracellular sorting, and processing of exosomes by dendritic cells. Blood 104, 3257-3266 (2004).

22. Thery, C. et al. Proteomic analysis of dendritic cell-derived exosomes: a secreted subcellular compartment distinct from apoptotic vesicles. J Immunol 166, 7309-7318 (2001).

23. Ramalho, J.S. et al. Chromosomal mapping, gene structure and characterization of the human and murine RAB27B gene. BMC Genet 2, 2 (2001).

24. Thery, C., Amigorena, S., Raposo, G. \& Clayton, A. Isolation and characterization of exosomes from cell culture supernatants and biological fluids. Curr Protoc Cell Biol Chapter 3, Unit 322 (2006).

25. Desnos, C. et al. Myosin va mediates docking of secretory granules at the plasma membrane. J Neurosci 27, 10636-10645 (2007).

26. Huet, S. et al. Analysis of transient behavior in complex trajectories: application to secretory vesicle dynamics. Biophys J 91, 3542-3559 (2006).

27. Nofal, S., Becherer, U., Hof, D., Matti, U. \& Rettig, J. Primed vesicles can be distinguished from docked vesicles by analyzing their mobility. $J$ Neurosci 27, 13861395 (2007).

28. Beraud-Dufour, S. \& Balch, W. A journey through the exocytic pathway. J Cell Sci 115, 1779-1780 (2002).

29. Pereira-Leal, J.B. \& Seabra, M.C. Evolution of the Rab family of small GTP-binding proteins. J Mol Biol 313, 889-901 (2001).

30. Buschow, S.I. et al. MHC II in Dendritic Cells is Targeted to Lysosomes or T CellInduced Exosomes Via Distinct Multivesicular Body Pathways. Traffic 10, 1528-1542 (2009).

31. Desnos, C. et al. Rab27A and its effector MyRIP link secretory granules to F-actin and control their motion towards release sites. J Cell Biol 163, 559-570 (2003).

32. Chen, X. et al. Rab27b localizes to zymogen granules and regulates pancreatic acinar exocytosis. Biochem Biophys Res Commun 323, 1157-1162 (2004).

33. Imai, A., Yoshie, S., Nashida, T., Shimomura, H. \& Fukuda, M. The small GTPase Rab27B regulates amylase release from rat parotid acinar cells. J Cell Sci 117, 19451953 (2004). 
34. Mizuno, K. et al. Rab27b regulates mast cell granule dynamics and secretion. Traffic 8, 883-892 (2007).

35. Tolmachova, T., Abrink, M., Futter, C.E., Authi, K.S. \& Seabra, M.C. Rab27b regulates number and secretion of platelet dense granules. Proc Natl Acad Sci U S A 104, 5872-5877 (2007).

36. Stinchcombe, J.C. et al. Rab27a is required for regulated secretion in cytotoxic T lymphocytes. J Cell Biol 152, 825-834 (2001).

37. Barral, D.C. et al. Functional redundancy of Rab27 proteins and the pathogenesis of Griscelli syndrome. J Clin Invest 110, 247-257 (2002).

38. Strom, M., Hume, A.N., Tarafder, A.K., Barkagianni, E. \& Seabra, M.C. A family of Rab27-binding proteins. Melanophilin links Rab27a and myosin Va function in melanosome transport. J Biol Chem 277, 25423-25430 (2002).

39. Seabra, M.C. \& Coudrier, E. Rab GTPases and myosin motors in organelle motility. Traffic 5, 393-399 (2004).

40. Savina, A., Vidal, M. \& Colombo, M.I. The exosome pathway in K562 cells is regulated by Rab11. J Cell Sci 115, 2505-2515 (2002).

41. Kondo, H. et al. Constitutive GDP/GTP exchange and secretion-dependent GTP hydrolysis activity for Rab27 in platelets. J Biol Chem 281, 28657-28665 (2006).

42. Stumptner-Cuvelette, P. et al. HIV-1 Nef impairs MHC class II antigen presentation and surface expression. Proc Natl Acad Sci U S A 98, 12144-12149 (2001).

43. Hume, A.N. et al. Rab27a regulates the peripheral distribution of melanosomes in melanocytes. J Cell Biol 152, 795-808 (2001).

44. Blott, E.J., Bossi, G., Clark, R., Zvelebil, M. \& Griffiths, G.M. Fas ligand is targeted to secretory lysosomes via a proline-rich domain in its cytoplasmic tail. J Cell Sci 114, 2405-2416 (2001).

45. Fukuda, M., Kanno, E., Saegusa, C., Ogata, Y. \& Kuroda, T.S. Slp4-a/granuphilin-a regulates dense-core vesicle exocytosis in PC12 cells. J Biol Chem 277, 39673-39678 (2002).

46. Shu, X., Shaner, N.C., Yarbrough, C.A., Tsien, R.Y. \& Remington, S.J. Novel chromophores and buried charges control color in mFruits. Biochemistry 45, 96399647 (2006).

47. Moffat, J. et al. A lentiviral RNAi library for human and mouse genes applied to an arrayed viral high-content screen. Cell 124, 1283-1298 (2006).

48. Racine, V. et al. Visualization and quantification of vesicle trafficking on a threedimensional cytoskeleton network in living cells. J Microsc 225, 214-228 (2007).

49. Steyer, J.A. \& Almers, W. Tracking single secretory granules in live chromaffin cells by evanescent-field fluorescence microscopy. Biophys J 76, 2262-2271 (1999). 


\section{Supplementary Informations :}

Supplementary Figure S1: Semi-quantitative detection of OVA in cell culture supernatants. (a) Representative flow cytometry dot-plots showing the detection of OVA present in the supernatants collected from cultures of increasing numbers of cells, after capture onto anti-OVA-coated beads and staining with Alexa488-anti-OVA Ig. (b) Doseresponse curve of OVA staining, obtained by quantification of 66 experimental conditions. (c) Comparison of the signal obtained following OVA staining in supernatants collected from cultures of either live or UV-irradiated cells collected $24 \mathrm{~h}$ after seeding 50,000 cells/well in a 96-well plate.

\section{Supplementary Figure S2: Further characterization of cells stably transduced with} Rab27a- or Rab27b-specific shRNAs. (a) Specific extinction of $R A B 27 A$ (respectively $R A B 27 B$ ) gene expression by Rab27a-specific virus 3 (respectively Rab27b-specific virus 1 ), with no effect on $R A B 27 B$ (respectively $R A B 27 A$ ) gene expression, as shown by quantitative RT-PCR with primers specific for each gene. (b) Representative dot plot showing HLADR/CD81 (top panel) and Annexin V (bottom panel) staining on large-scale supernatants from cells transduced with Rab27a-specific virus 3 and Rab27b-specific virus 1.

Supplementary Figure S3: Morphology of MVEs in the subplasmalemmal region. (a) Colocalisation of GFP-Rab27a or GFP-Rab27b and CD63 (labeled with anti-mouse Cy3) observed by TIRFM. Quantification of colocalisation was performed on 10 cells per condition. Scale bar: $5 \mu \mathrm{m}$. Zoom: x2.5. (b) Rab27a and Rab27b silencing increase the distance of the vesicles to the border of the cells. The areas of the cell footprints (yellow), and of the minimal polygon encompassing all vesicles (red) were measured. Representative images of control, Rab27a KD and Rab27b KD cell are shown. The ratio of the vesicles area / total cell area was calculated in 23 control cells, 23 Rab27a KD cells and 25 Rab27b KD cells. (c) GFP-CD63 vesicles were tracked and diffusion coefficients $D_{x y}$ were computed along the trajectories. Shown is the distribution of $D_{x y}$ pooled from 118 trajectories monitored in 7 control cells. Values above $30 \times 10^{-4} \mu^{2} . \mathrm{s}^{-1}$ were omitted. An example of a vesicle 
exhibiting a directed motion (0-5 s) followed by a docking period (5-10 s, 15-25 s) is presented in the inset.

\section{Supplementary video-movies:}

Video 1 for a CTRL cell, video 2 for a Rab27a KD cell and Video 3 for a Rab27b KD cell. Images were acquired by TIRFM at $10 \mathrm{~Hz}$ for $40 \mathrm{~s}$. Movies are accelerated 4 times.

\section{Supplementary Figure S4: Effect of double-silencing of Rab27a or Rab27b and Slp4 on the morphology of CD63-positive MVEs.}

B6H4 stably expressing shRNA to either Rab27a or Rab27b were transfected with a plasmid encoding EGFP and a shRNA to Slp4. Four days later, cells were fixed and stained with antiCD63, and morphology of CD63 compartments was manually quantified in GFP-expressing cells. (a) Quantification of cells presenting enlarged CD63+ compartments. Single KD of Rab27a and double KD of Rab27a and Slp4 induce the same phenotype (enlarged MVEs). (b) Quantification of cells presenting asymmetrical perinuclear accumulation of CD63+ compartments. Slp4 KD does not induce asymmetrical distribution of MVEs, whereas both single KD of Rab27b and double Rab27b+Slp4 KD induce this phenotype. For each condition, 20 cells were evaluated in a blind manner.

Supplementary Figure S5: Exosome secretion by bone marrow-derived dendritic cells (BMDCs) from Rab27a/Rab27b double knock-out mice.

Dendritic cells were differentiated from bone marrow precursors in the presence of GM-CSF. At day 10 after the onset of culture, cells were cultured in exosome-depleted medium for 24h, before collection of supernatant and purification of exosomes by ultracentrifugation. WT and Rab27a/b-KO cells contained more than $75 \%$ of BMDCs, and less than $10 \%$ of mature BMDCs, as assessed by FACS staining for CD11c, Gr1, MHC Class II. Purified exosomes secreted by equal amounts of control or KO cells were analysed by immunoblotting for presence of CD9, tsg101, MFG-E8, MHC class II, and gp96 proteins. A total of 30 or $10 \mu \mathrm{g}$ cells were lysed and used for comparison. One representative experiment of four is shown. Histogram shows quantification of signal intensity of CD9, tsg101, MFG-E8, and MHC II proteins in exosomes secreted by 5.8 million cells from the KO mice, normalized to the value obtained with exosomes from control cells (mean \pm SD of 4 experiments). 
Supplementary Figure S6: Uncropped Western blots corresponding to Figures 3b, 3c, 7c, 8b, and 7d.

Western blots corresponding to Figures 3b (a), 3c (b), 7c (c), 7d (e) and 8b (d) were hybridized consecutively with antibodies in the displayed order. Position of molecular weight markers $(\mathrm{kDa})$ is indicated. Bands corresponding to the specific protein are highlighted by an arrow. 